\title{
Throughput Analysis for the Cognitive Uplink Under Limited Primary Cooperation
}

\author{
Ehsan Nekouei, Member, IEEE, Hazer Inaltekin, Member, IEEE, and Subhrakanti Dey, Senior Member, IEEE
}

\begin{abstract}
This paper studies the achievable throughput performance of the cognitive uplink under a limited primary cooperation scenario wherein the primary base station cannot feed back all interference channel gains to the secondary base station. To cope with the limited primary cooperation, we propose a feedback protocol called $K$-out-of- $N$ feedback protocol, in which the primary base station feeds back only the $K_{N}$ smallest interference channel gains, out of $N$ of them, to the secondary base station. We characterize the throughput performance under the $K$-out-of- $N$ feedback protocol by analyzing the achievable multiuser diversity gains (MDGs) in cognitive uplinks for three different network types. Our results show that the proposed feedback mechanism is asymptotically optimum for interference-limited (IL) and individual-power-and-interferencelimited (IPIL) networks for a fixed positive $K_{N}$. It is further shown that the secondary network throughput in the IL and IPIL networks (under both the full and limited cooperation scenarios) logarithmically scales with the number of users in the network. In total-power-and-interference-limited (TPIL) networks, on the other hand, the $K$-out-of- $N$ feedback protocol is asymptotically optimum for $K_{N}=N^{\delta}$, where $\delta \in(0,1)$. We also show that, in TPIL networks, the secondary network throughput under both the limited and full cooperation scales logarithmically double with the number of users in the network. These results indicate that the cognitive uplink can achieve the optimum MDG even with limited cooperation from the primary network. They also establish the dependence of pre-log throughput scaling factors on the distribution of fading channel gains for different network types.
\end{abstract}

Index Terms-Cognitive radio, multiple-access networks, multiuser diversity, throughput scaling, channel state feedback.

\section{INTRODUCTION}

\section{A. Background and Motivation}

$\mathbf{C}$ OGNITIVE RADIO technology has emerged as a revolutionary suite of communication techniques in response to the sheer pressure of having a more dynamic

Manuscript received September 22, 2015; revised February 27, 2016; accepted May 3, 2016. Date of publication May 13, 2016; date of current version July 12, 2016. This research was supported in part by the Scientific and Technological Research Council of Turkey (TUBITAK) under Grant 112E024 and in part by the European Commission Research Executive Agency Marie Curie FP7-Reintegration-Grants under Grant PCIG10-GA-2011-303713. This paper was presented at the IEEE Global Communications Conference, Anaheim, California, December 2012. The associate editor coordinating the review of this paper and approving it for publication was H. R. Bahrami.

E. Nekouei is with the Department of Electrical and Electronic Engineering, The University of Melbourne, Melbourne, VIC 3010, Australia (e-mail: ehsan.nekouei@unimelb.edu.au).

H. Inaltekin is with the Department of Electrical and Electronics Engineering, Antalya International University, Antalya 07190, Turkey (e-mail: hazeri@antalya.edu.tr).

S. Dey is with the Division of Signals and Systems, Uppsala University, Uppsala 75121, Sweden (e-mail: subhra.dey@signal.uu.se).

Color versions of one or more of the figures in this paper are available online at http://ieeexplore.ieee.org.

Digital Object Identifier 10.1109/TCOMM.2016.2568200 means of managing spectrum and exploiting likely spectrum holes [1]-[3]. Roughly speaking, it alleviates the spectrum scarcity problem by allowing unlicensed third parties (alternatively called: secondary users or SUs) to share the same bandwidth with the incumbent users (alternatively called: primary users or PUs) provided that their transmissions do not cause harmful degradation to the primary transmission.

To this end, a secondary network requires the knowledge of interference channel gains from its SUs to the primary basestation (PBS) to successfully keep the secondary interference power at the primary network below pre-determined critical levels. Hence, interference channel gains pertaining to SUs need to be conveyed to the secondary base-station (SBS) by means of a primary-secondary feedback link, e.g., see the papers [4]-[6] that require full primary cooperation. However, it is not always possible or even desirable to deploy high capacity feedback links between PBS and SBS due to the high deployment cost of feedback links, especially for $4 \mathrm{G}$ networks and beyond, e.g., see the papers [7]-[9] and the references therein. Therefore, it becomes almost impractical for the PBS to convey all interference channel gains to the SBS within the channel coherence time over low-cost feedback links lacking sufficient communication resources for high data rate transmission.

One remedy for the primary-secondary feedback problem is to design cognitive radio networks that are able to operate without utilizing primary-secondary feedback links, i.e., no primary cooperation at all. Recently, the authors in [10] proposed a distributed power control mechanism that completely eliminates the necessity of implementing the primarysecondary feedback link for cognitive uplinks, albeit with some loss in the data rates. In the proposed approach, each SU measures/estimates its interference and power channel gains locally, and independently decides to transmit based on the quality of measured channel gains. The throughput analysis in [10] shows that the distributed cognitive uplink suffers from a drastic reduction in the achievable throughput (i.e., more than $60 \%$ ).

These recent findings motivate the current paper to obtain alternative solutions to alleviate the feedback problem for cognitive radio multiple-access networks without reducing secondary communication rates. In particular, our main research questions can be stated as: $(i)$ Can a cognitive radio uplink with limited primary cooperation achieve a throughput performance close to that of cognitive uplinks with full primary cooperation?, $(\mathrm{ii})$ what is the structure of optimum or asymptotically optimum primary-secondary feedback protocol for a cognitive uplink? In this paper, we consider a cognitive uplink in which the PBS is only able to feed back 
a limited number of interference channel gains to the SBS due to capacity limitations of the feedback link between them. We propose a primary-secondary feedback protocol that substantially reduces the amount of required feedback exchange between PBS and SBS. We evaluate the efficacy of the proposed feedback protocol by analyzing its achievable multiuser diversity gains (MDGs) for different network types.

Our results show that, under the proposed feedback protocol, a cognitive uplink can achieve throughput performance similar to that of cognitive uplinks with full primary cooperation. These results are derived for a parametrized family of general fading distributions called class $C$-distributions. The available tools in the literature for analyzing MDG in cognitive radio networks are applicable only if one can find a closed form expression for the joint channel states. This is not always possible when direct and interference channel gains are arbitrarily distributed. One particular example is when direct and interference channel gains are Nakagami- $m$ distributed. Hence, dealing with a parametrized family of distributions to derive generalized MDGs requires an investigation of more subtle concentration behavior of extreme order statistics to obtain tail estimates of joint channel states, which is technically much more challenging than assuming specific distributions for direct and interference channel gains.

In addition to being technically challenging, perhaps more importantly, our analysis provides new insights into the network operation by relating the fading distribution parameters to the pre-log factors in the derived throughput scaling laws, which is otherwise hidden by assuming specific fading processes such as Rayleigh fading.

\section{B. Main Contributions}

In this paper, we consider two primary-secondary feedback scenarios: $(i)$ full cooperation scenario and (ii) limited cooperation scenario. In the full cooperation scenario, the PBS conveys all the interference channel gains to the SBS, i.e., no limitation is considered on the capacity of feedback link. In the limited cooperation scenario, the PBS only feeds back $K_{N}$ smallest interference channel power gains to the SBS. $K_{N}$ can be interpreted as our modelling parameter to numerically designate the feedback capability of the backhaul link. We refer to this feedback policy as the $K$-out-of- $N$ feedback protocol.

We focus on three types of secondary networks, and for each network type considered, we provide a sufficient condition on $K_{N}$ such that the $K$-out-of- $N$ feedback protocol is asymptotically optimum, i.e., it achieves the same MDG as that of full cooperation scenario. We allow the distributions of direct and interference channel gains to be arbitrarily selected from the class- $C$ distributions (i.e., see Definition 3.1). The class- $C$ distributions include Rayleigh, Rician, Nakagami and Weibull fading models as special cases. This allows us to derive the generalized MDGs for cognitive uplinks with full/limited primary cooperation.

Network types considered are: Interference-Limited (IL), Individual-Power-and-Interference-Limited (IPIL) and TotalPower-and-Interference-Limited (TPIL) networks. In IL, we show that the $K$-out-of- $N$ feedback protocol is asymptotically optimum for $K_{N}=O(1)$. Moreover, our results show that, in the IL networks, the secondary network throughput under both full and limited primary cooperation scenarios scales according to $\frac{1}{\gamma_{g}} \log (N)$, where $\gamma_{g}$ is a parameter determined from the behavior of the cumulative distribution function (CDF) of the interference channel power gains around zero. (see Definition 3.1 and Table II for more information about $\gamma_{g}$.) Similar results continue to hold for the IPIL networks. In particular, our results show that the $K$-out-of$N$ feedback protocol is asymptotically optimum for $K_{N}=$ $O(1)$, while the secondary network throughput under both full and limited primary cooperation scales according to $\min \left(1, \frac{1}{\gamma_{g}}\right) \log (N)$.

In TPIL networks, on the other hand, our results indicate that the $K$-out-of- $N$ feedback protocol is asymptotically optimum for $K_{N}=N^{\delta}$, where $0<\delta<1$ For $K_{N}=N^{\delta}$, the secondary network throughput in the TPIL networks scales according to $\frac{1}{n_{h}} \log \log (N)$ under both full and limited primary cooperation scenarios, where $n_{h}$ is a parameter determined from the asymptotic tail behavior of direct channel power gains. (see Definition 3.1 and Table II for more information about $n_{h}$.) In TPIL networks, we further show that, for $K_{N}=o(N)$, the interference power at the PBS converges to zero almost surely and also in mean as $N$ tends to infinity. From a practical point of view, this result implies that the interference constraint is not a performance limiting criterion for $N$ large enough. Hence, the SBS just requires the indices of the SUs with the lowest $K_{N}$ interference channel gains, rather than the actual realizations of the interference channel gains, which provides an added reduction on the amount of feedback load required between two networks. Our main results are summarized in Table I.

\section{A Note on Notation and Paper Organization}

When we write $p(x)=O(q(x))$ and $p(x)=o(q(x))$ for two positive functions $p(x)$ and $q(x)$, we mean $\lim \sup _{x \rightarrow \infty} \frac{p(x)}{q(x)}<\infty$ and $\lim _{x \rightarrow \infty} \frac{p(x)}{q(x)}=0$, respectively. By $p(x)=\Theta(q(x))$, we mean $0<\liminf _{x \rightarrow \infty} \frac{p(x)}{q(x)} \leq$ $\limsup _{x \rightarrow \infty} \frac{p(x)}{q(x)}<\infty$.

As is standard in the literature, when we say a wireless channel is a Rayleigh fading channel, we mean that the channel magnitude gain is Rayleigh distributed, or equivalently the channel power gain is exponentially distributed. By a Rician- $k$ fading channel, we mean that the channel magnitude gain is Rician distributed with a Rician factor $k \geq 0$. For a Rician- $k$ fading channel, the channel power gain is non-central chi-square distributed with two degrees of freedom [11]. When we say a wireless channel is Nakagami- $m$ distributed, we mean that the channel magnitude gain is Nakagami distributed with a Nakagami factor $m \geq 0.5$. For a Nakagami- $m$ fading channel, the channel power gain is Gamma distributed. By a Weibull- $c$ fading channel, we mean that the channel magnitude gain is Weibull distributed with a Weibull parameter $c>0$. We refer the reader to [11] and [12] for more details about fading distributions. 
TABLE I

Throughrut ScAling LaWs For DifFerent Network Models

\begin{tabular}{lcc}
\hline \multirow{2}{*}{ Network Model } & \multicolumn{2}{c}{ Feedback Protocol } \\
\cline { 2 - 3 } & $\lim _{N \rightarrow \infty} \frac{R_{N}{ }^{a}}{\log (N)}=\frac{1}{\gamma_{g}}{ }^{b}$ & $\lim _{N \rightarrow \infty} \frac{R_{N}}{\log (N)}=\frac{1}{\gamma_{g}}$ \\
\hline $\begin{array}{l}\text { Interference-Limited } \\
\left(K_{N}=O(1)\right)\end{array}$ & $\lim _{N \rightarrow \infty} \frac{R_{N}}{\log (N)}=\min \left(1, \frac{1}{\gamma_{g}}\right)$ & $\lim _{N \rightarrow \infty} \frac{R_{N}}{\log (N)}=\min \left(1, \frac{1}{\gamma_{g}}\right)$ \\
\hline $\begin{array}{l}\text { Individual-Power-And-Interference-Limited } \\
\left(K_{N}=O(1)\right)\end{array}$ & $\lim _{N \rightarrow \infty} \frac{R_{N}}{\log \left(\log \left(K_{N}\right)\right)}=\frac{1}{n_{h}} c$ & $\lim _{N \rightarrow \infty} \frac{R_{N}}{\log (\log (N))}=\frac{1}{n_{h}}$ \\
\hline Total-Power-And-Interference-Limited & & \\
$\left(K_{N}=o(N)\right)$ & & \\
\hline
\end{tabular}

${ }^{a} R_{N}$ is the secondary network aggregate throughput.

${ }^{b} \gamma_{g}$ is a parameter determined from the behavior of the CDF of interference channel power gains around the origin.

${ }^{c} n_{h}$ is a parameter determined from the asymptotic tail behavior of the CDF of direct channel power gains.

The rest of the paper is organized as follows. In Section II, we discuss the relevant literature. Section III describes the system model and network configuration along with our modeling assumptions. Section IV derives and compares the secondary network throughput scaling under $K$-out-of- $N$ and full primary cooperation scenarios, discusses the effect of fading channel parameters on the scaling laws and provides various insights into the derived throughput scaling laws by focusing on the constant primary interference power at the SBS. Section V extends the results presented in Section IV to the case of randomly varying primary's interference power. Section VI illustrates the accuracy of our results by means of numerical examples. Section VII concludes the paper and presents some future research directions of interest. All proofs are relegated to appendices and the associated technical report [27] due to space limitations.

\section{RELATED WORK}

The jointly optimum power control and spectrum sharing problem under limited primary cooperation has been previously studied in the cognitive radio literature in recent papers such as [10], [13]-[15], and [26]. The authors in [13] studied the optimal power control problem for a secondary transmitterreceiver pair sharing a frequency band with a primary receiver. In their set-up, the secondary transmitter has only access to the quantized channel state information (CSI) of the primary link and the transmission power of the SU is limited by an average transmission power constraint and the primary rate loss constraint. The authors in [14] considered a setup similar to [13], and studied the optimal power control problem when the secondary transmitter receives the quantized version of its direct channel gain as well as the quantized channel state information of the primary link. In [15], the authors considered the optimal power control problem in a cognitive broadcast channel in which the SBS receives onebit information about the quality of the primary link and onebit information about the quality of each secondary link. They established the double logarithmic capacity scaling law for the secondary network under the optimal power control policy at the SBS for Rayleigh distributed fading channel gains. Similar to these previous works, our performance measure in this paper is also the secondary network throughput capacity under the jointly optimum power control and spectrum sharing policy. Different from them, we focus on cognitive uplinks wherein many SUs interfere with the primary's transmission, and establish the capacity scaling laws under limited primary cooperation for a generalized fading model.

The reference [10] only reports the throughput scaling results in fully distributed cognitive uplinks, and the case of reduced primary cooperation was not considered in [10]. In particular, the capacity scaling results with distributed scheduling are quite restrictive due to an extra pre-log multiplier of $\frac{1}{\mathrm{e}}$. On the other hand, the proposed $K$-out-of- $N$ feedback protocol has the potential to boost the capacity performance of cognitive radio networks three times by means of small feedback load coordinating SU transmissions. From an engineering point of view, this result leads to a critical design choice for network operators, to deploy or not to deploy a low capacity feedback link between primary and secondary networks, to improve data rates in next generation cognitive radio empowered telecommunication technologies. From a more technical point of view, the channel model and the operating constraints in [10] are significantly different than those in this paper. Specifically, a collision channel model is employed in [10] due to distributed implementation, whereas a classical Gaussian multiple-access channel model with successive interference cancellation is used in the current paper. Furthermore, the structure of the optimum power control policy derived in [10], which is a threshold-based power control policy, is also different from the structure of the optimum power control policy derived here. These distinctions manifest themselves as fundamental differences in the proof techniques to establish capacity scaling laws in both cases. In [26], which is the conference version of this work, the capacity scaling behavior of TPIL networks under full and $K$-out-of- $N$ feedback protocols is presented. The capacity scaling behavior of IPIL and IL networks under full and $K$-out-of- $N$ feedback protocols as well as the required 
conditions on $K_{N}$ for asymptotic optimality of these scaling results were not reported in [26]. It should be emphasized that the results for IL and IPIL networks are structurally much different than those in [26] and cannot be deduced from the results for TPIL networks.

Capacity scaling laws for the cognitive uplink under full CSI have also been investigated in the literature, e.g., see [16]-[21], under various types of constraints on the transmission powers of SUs. In [16], the authors studied MDGs in an interweave cognitive uplink wherein SUs report their decisions about primary's activity to the SBS. Then, based on the received information from SUs, the SBS schedules the best SU for transmission. The authors in [17] and [18] studied the capacity scaling laws for Rayleigh fading cognitive multiple access networks under joint peak transmission and interference power constraints. For fixed/adaptive power control policies regulating SUsÕ transmissions, they established logarithmic and double logarithmic secondary network capacity scaling behavior through some approximations. These results are extended to cognitive broadcast and cognitive parallel access channels in [19]. The authors in [20] considered a cognitive uplink sharing spectrum with a primary broadcast network. Assuming Rayleigh fading channels, they analyzed MDGs for the secondary network under a fixed transmission power policy. In [21], the authors studied throughput scaling behavior of IL and TPIL cognitive multiple access networks under the full CSI assumption when transmission powers of SUs are optimally allocated. The current work differs from these previous papers in that [16]-[21] do not consider feedback limited communication environments, and assume specific fading distributions to derive the stated throughput capacity scaling laws. Further, using a generalized class of fading distributions, this paper establishes the connection between the pre-log factors in the capacity scaling results and the distribution of fading channels, which does not appear in these previous studies.

Other related work includes secondary network capacity scaling in multi-band and multi-antenna setups such as [22], [23] and [24]. In [22], the authors studied the multiuser and multi-spectrum diversity gains for a cognitive broadcast network sharing multiple orthogonal frequency bands with a primary network. Assuming Rayleigh fading channels and peak interference power limitations, they derived capacity scaling expressions for the secondary network. In [23], the authors considered $N$ secondary transmitter-receiver pairs sharing $M$ frequency bands with a primary network. Under the optimum matching of SUs with primary network frequency bands, they obtained a double logarithmic capacity scaling law for Rayleigh fading secondary networks. In [24], the authors studied the scheduling gain in cognitive radio networks with multiple antennas at the SBS and PBS. They established logarithmic throughput scaling laws for the secondary network with pre-log multipliers depending on the operating modes (i.e., multiple access versus broadcast) and the number of antennas at the SBS and PBS. When compared to [22], [23], and [24], this paper differs from them in three important aspects. First, we focus on more practical feedback limited communication scenarios. Second, all of our results are derived

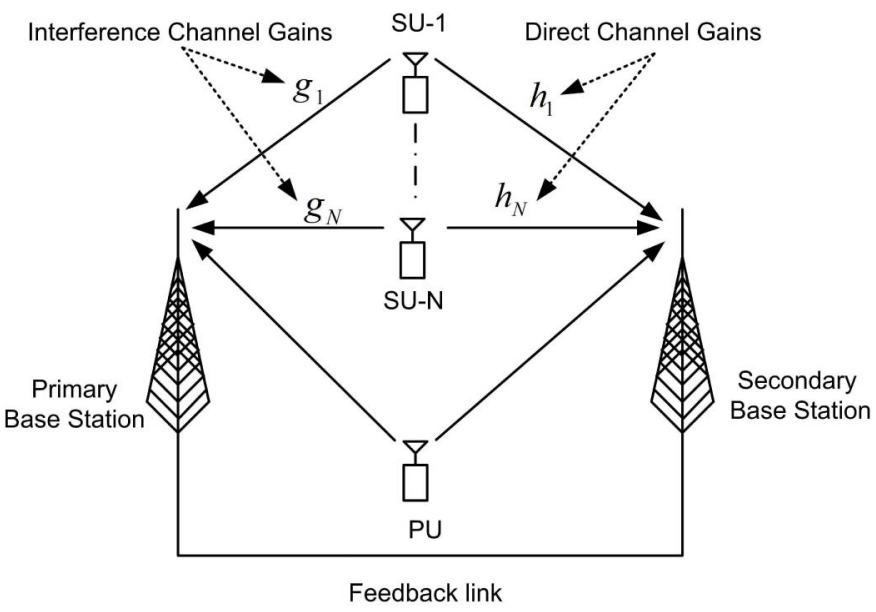

Fig. 1. $\quad N$ SUs forming a multiple access channel to the SBS and interfering with signal reception at the PBS. The backhaul feedback link can be implemented by using either a microwave link or a DSL link with limited capacity.

under optimum allocation of transmission powers to SUs. Third, by considering general fading models, we establish generalized MDGs in cognitive uplinks under full and limited primary cooperation scenarios. These differences result in using more subtle concentration behavior of extreme order statistics to obtain tail estimates of joint channel states as put forward in Appendices A and B.

\section{System Model, Operating Constraints AND THE NETWORK TYPES}

In this section, we will introduce the details of our system model, the operating constraints on the cognitive radio environment that go with this model and the classification of the network types studied throughout the paper based on these operating constraints.

\section{A. System Model}

We consider an underlay cognitive uplink in which $N$ SUs transmit data to an SBS and interfere with the signal reception at a PBS. Let $h_{i}$ and $g_{i}$ represent the fading power gains for the $i$ th direct and interference links, respectively. The classical ergodic block fading model [25] is assumed to hold to model statistical variations in channel states for all direct and interference links. Further, we assume that $h_{i}$ 's are independent and identically distributed (i.i.d.) random variables among themselves and $g_{i}$ 's are i.i.d. random variables among themselves, but direct channel gains $h_{i}, i=1, \ldots, N$, may have a different joint distribution than that of interference channel gains $g_{i}, i=1, \ldots, N$. That is, the random vectors $\boldsymbol{h}=\left[h_{1}, h_{2}, \ldots, h_{N}\right]^{\top}$ and $\boldsymbol{g}=\left[g_{1}, g_{2}, \ldots, g_{N}\right]^{\top}$ are also independent, but possibly with different distributions. The explained communication set-up is represented in Fig. 1 pictorially.

To describe the direct and interference channel variations over time, we consider a general class of parametrized distributions, which is formally introduced in the following definition. 
TABLE II

Common Fading Channel Models and Their Parameters

\begin{tabular}{|c|c|c|c|c|c|c|c|}
\hline \multirow{2}{*}{ Channel Model } & \multicolumn{7}{|c|}{ Parameters } \\
\hline & $\alpha$ & $l$ & $\beta$ & $n$ & $H(x)$ & $\eta$ & $\gamma$ \\
\hline Rayleigh & 1 & 0 & 1 & 1 & 0 & 1 & 1 \\
\hline Rician- $k$ & $\frac{1}{2 \sqrt{\pi} \mathrm{e}^{k} \sqrt[4]{k(k+1)}}$ & $-\frac{1}{4}$ & $k+1$ & 1 & $2 \sqrt{k(k+1) x}$ & $\frac{k+1}{\mathrm{e}^{k}}$ & 1 \\
\hline Nakagami- $m$ & $\frac{m^{m-1}}{\Gamma(m)}$ & $m-1$ & $m$ & 1 & 0 & $\frac{m^{m-1}}{\Gamma(m)}$ & $m$ \\
\hline Weibull- $c$ & 1 & 0 & $\Gamma^{\frac{c}{2}}\left(1+\frac{2}{c}\right)$ & $\frac{c}{2}$ & 0 & $\Gamma^{\frac{c}{2}}\left(1+\frac{2}{c}\right)$ & $\frac{c}{2}$ \\
\hline
\end{tabular}

Definition 3.1: We say that the CDF of a random variable $X$, denoted by $F_{X}$, belongs to the class- $C$ distributions if it satisfies the following properties:

- $F_{X}(x)$ is continuous.

- $F_{X}(x)$ has a positive support, i.e., $F(x)=0$ for $x \leq 0$.

- $F_{X}(x)$ is strictly increasing.

- The tail function $1-F(x)$ decays to zero exponentially, i.e., there exist constants $\alpha>0, \beta>0, n>0, l \in \mathbb{R}$ and a slowly varying function $H(x)$ satisfying $H(x)=o\left(x^{n}\right)$ such that $\lim _{x \rightarrow \infty} \frac{1-F(x)}{\alpha x^{l} \mathrm{e}\left(-\beta x^{n}+H(x)\right)}=1$.

- $F(x)$ varies regularly around the origin, i.e., there exist constants $\eta>0$ and $\gamma>0$ such that $\lim _{x \rightarrow 0} \frac{F(x)}{\eta x^{\gamma}}=1$.

Our results in Theorems 1, 2 and 3 in Section IV indicate that the channel gain distribution parameters play important roles in identifying the pre-log factor in the fundamental capacity scaling laws for cognitive radio networks. In particular, the decay rate of the CDF around zero and that of its associated tail function around infinity determine the nature of full MDG, which is otherwise hidden by only considering the Rayleigh fading scenario. We will elaborate on these findings further as we discuss the above theorems in Section IV. The parameters characterizing the behavior of the distribution of fading power gains around zero and infinity are illustrated in Table II for the commonly used fading models in the literature. To avoid any confusion, we represent these parameters with subscript $h$ for direct channel gains and with subscript $g$ for interference channel gains, e.g., $\eta_{g}$ or $\eta_{h}$, in the remainder of the paper.

\section{B. Operating Constraints}

We consider different operating constraints on the cognitive radio environment introduced above in order to identify the network types studied throughout the paper more systematically. Specifically, different constraints on the transmission powers of SUs and the capacity of the feedback link are considered. In each case, we analyze the throughput scaling behavior of the secondary network when the transmission powers of SUs are allocated according to an optimum power allocation policy subject to these constraints, where we define a power allocation policy $\boldsymbol{P}(\boldsymbol{h}, \boldsymbol{g})=$ $\left[P_{1}(\boldsymbol{h}, \boldsymbol{g}), \cdots, P_{N}(\boldsymbol{h}, \boldsymbol{g})\right]^{\top}$ as a mapping from $\mathbb{R}^{2 N}$ to $\mathbb{R}^{N}$ in which $P_{i}(\boldsymbol{h}, \boldsymbol{g})$ is the transmission power of the $i$ th $\mathrm{SU}$.

The equations (1a), (1b), (1c), (1d) and (1e) below list the operating constraints on the studied cognitive radio environment formally, and the throughput scaling behavior of the secondary networks is derived systematically under different combinations of these constraints. In particular, (1a), (1b) and (1c) are average total power, average individual power and average total interference power constrains, respectively. (1d) is a constraint to guarantee that a SU is allowed to transmit only if its interference channel gain is available at the SBS, where $g_{K_{N}: N}$ represents the $K_{N}$ th smallest value in the set $\left\{g_{i}\right\}_{i=1}^{N}$. (1d) will be called the feedback constraint in the remainder of the paper as it describes the SUs that are allowed for transmission as a function of the feedback load $K_{N}$. Equation (1e) is the usual positivity constraint on the transmission power, which is added for the sake of mathematical completeness. ${ }^{1}$

$$
\left\{\begin{array}{l}
\mathrm{E}_{\boldsymbol{h}, \boldsymbol{g}}\left[\mathbf{1}^{\top} \boldsymbol{P}(\boldsymbol{h}, \boldsymbol{g})\right] \leq P_{\mathrm{ave}}, \\
\mathrm{E}_{\boldsymbol{h}, \boldsymbol{g}}\left[P_{i}(\boldsymbol{h}, \boldsymbol{g})\right] \leq P_{\mathrm{ave}} \quad 1 \leq i \leq N, \\
\mathrm{E}_{\boldsymbol{h}, \boldsymbol{g}}\left[\boldsymbol{g}^{\top} \boldsymbol{P}(\boldsymbol{h}, \boldsymbol{g})\right] \leq Q_{\mathrm{ave}}, \\
P_{i}(\boldsymbol{h}, \boldsymbol{g}) 1_{\left\{g_{i} \geq g_{K_{N}: N}\right\}}=0 \quad 1 \leq i \leq N, \\
P_{i}(\boldsymbol{h}, \boldsymbol{g}) \geq 0 .
\end{array}\right.
$$

From an implementation point of view, the PBS needs to rank the interference channel power gains and select the $K_{N}$ smallest ones to execute the $K$-out-of- $N$ feedback protocol. The computational complexity of this ordering process is only polynomial in $N$. Further, a negotiation process may take place between primary and secondary networks to reach an agreement on the final value of $K_{N}$ before the commencement of the data communication phase. This has to be done only once, and hence the limited-capacity backhaul link between two networks can also be utilized for such ante-communication processes.

Remark 1: An important application scenario of the cognitive radio technology is the multi-tier heterogenous cellular networks in which multitude of cognitive radio devices are expected to opportunistically share the same spectrum with the incumbent cellular network users, e.g., see [28], [29], and references therein. In such heterogeneous communication environments, high capacity wired backhaul links are not

\footnotetext{
${ }^{1}$ Another important issue in this setup is the estimation of interference channel gains by the PBS. This can be efficiently done by using pilot signals transmitted intermittently by SUs. These pilot signals are heard by the PBS through interference channels, which can be further utilized to estimate interference channel gains.
} 
usually available to coordinate the transmissions of devices belonging to different network tiers. As a result, it turns out that the capacity of backhual links is a limiting factor in terms of cost and performance for multi-tier heterogenous cellular networks [30]. Thus, in these networks, the interference channel gains must be conveyed over capacity-limited wireless backhaul links, which necessitates the investigation of limited cooperation between PBS and SBS in this paper.

\section{TPIL Networks}

In TPIL networks, we examine the secondary network throughput scaling behavior in two communication scenarios (CoSs) of interest: $\mathrm{CoS}_{\mathrm{TPIL}}^{\text {Full }}$ and $\mathrm{CoS}_{\mathrm{TPIL}}^{K} \cdot \mathrm{CoS}_{\mathrm{TPIL}}^{\text {Full }}$ refers to a TPIL network under full cooperation scenario whereas $\operatorname{CoS}_{\text {TPIL }}^{K}$ refers to a TPIL network under $K$-out-of- $N$ feedback protocol. In $\mathrm{CoS}_{\mathrm{TPIL}}^{\mathrm{Full}}$, transmission powers of SUs are limited by an average total power constraint and an average total interference power constraint without any restriction on the amount of feedback information to be exchanged between the PBS and SBS. Hence, transmission powers of SUs are allocated according to the solution of the following optimization problem:

$\max _{\boldsymbol{P}(\boldsymbol{h}, \boldsymbol{g})} \mathrm{E}_{\boldsymbol{h}, \boldsymbol{g}}\left[\log \left(1+\frac{\boldsymbol{h}^{\top} \boldsymbol{P}(\boldsymbol{h}, \boldsymbol{g})}{W}\right)\right]$

subject to : (1a), (1c) and (1e),

where $W=N_{0}+I$ represents the average background noise plus primary interference power at the SBS. It is important to note here that our control actions are only on the SUs since we consider the underlay communication paradigm for the coexistence of primary and secondary networks [2]. Hence, the uncontrolled variable $W$ just functions as a scaling parameter for the secondary network direct channel gains. ${ }^{2}$

The solution to (2) was given in [21] as in (3), shown at the bottom of the page.

This result intuitively indicates that the jointly optimum spectrum sharing and power control policy maximizing information theoretic throughput capacity of a cognitive uplink with full primary cooperation under average total transmission and interference power constraints is to schedule the SU with the best joint direct and interference channel state summarized

\footnotetext{
${ }^{2}$ The joint control of primary and secondary networks is outside the scope of this paper. This will require a central authority that can oversee all control variables and channel gains, which implies excessive feedback load between primary and secondary networks and does not serve the purpose of throughput analysis under limited primary-secondary coordination. Further, we can also assume that the SBS can cancel primary interference to remove the coupling between primary and secondary networks as in [7].
}

by the random variable $X_{N}^{\star}\left(\lambda_{N}, \mu_{N}\right)=\max _{1 \leq i \leq N} \frac{h_{i} / W}{\lambda_{N}+\mu_{N} g_{i}}$ according to a power allocation policy in the form of a waterfilling algorithm with changing water levels. Here, $\lambda_{N}$ and $\mu_{N}$ are Lagrange multipliers associated with the average total transmission and interference power constraints, respectively. We note that there is no ambiguity with the solution described in (3) since direct and interference channel gains are continuous random variables, and there is only one SU achieving the maximum joint channel state with probability one. Let $R_{\mathrm{TPIL}}(N)$ be the throughput of the secondary network for the all feedback scenario. Then, it follows directly that

$$
R_{\mathrm{TPIL}}(N)=\mathrm{E}\left[\log \left(X_{N}^{\star}\left(\lambda_{N}, \mu_{N}\right)\right) 1_{\left.\left\{X_{N}^{\star}\left(\lambda_{N}, \mu_{N}\right) \geq 1\right\}\right]} .\right.
$$

Different from the full primary cooperation scenario, transmission powers of SUs in $\operatorname{CoS}_{\mathrm{TPIL}}^{K}$ are also limited by an extra feedback constraint given by (1d), besides the average total transmission and interference power constraints above. Hence, transmission powers of SUs in this case are allocated according to the solution of the following optimization problem:

$$
\max _{\boldsymbol{P}(\boldsymbol{h}, \boldsymbol{g})} \mathrm{E}_{\boldsymbol{h}, \boldsymbol{g}}\left[\log \left(1+\frac{\boldsymbol{h}^{\top} \boldsymbol{P}(\boldsymbol{h}, \boldsymbol{g})}{W}\right)\right]
$$$$
\text { subject to : (1a), (1c), (1d), and (1e). }
$$

Lemma 1: Let $\pi(j)$ be a mapping from $\left\{1, \cdots, K_{N}\right\}$ to $\{1, \cdots, N\}$ such that $\pi(j)=i$ if $g_{i}=g_{j: N}$. Then, the solution for (4) is given by (5), shown at the bottom of the page.

Proof: Follows directly by inspecting the structure of the solution given for (2) in (3).

As an analogy with the solution described in (3), the jointly optimum spectrum sharing and power allocation policy described in (5) under the limited primary cooperation is to schedule the SU with the best joint channel state among the ones that are fed back to the SBS. Specifically, the throughput in the $\operatorname{CoS}_{\mathrm{TPIL}}^{K}$ scenario can be written as

$$
R_{\mathrm{TPIL}}\left(K_{N}\right)=\mathrm{E}\left[\log \left(X_{K_{N}}^{\star}\left(\lambda_{N}, \mu_{N}\right)\right) 1_{\left\{X_{K_{N}}^{\star}\left(\lambda_{N}, \mu_{N}\right) \geq 1\right\}}\right] \text {, }
$$

where $X_{K_{N}}^{\star}\left(\lambda_{N}, \mu_{N}\right)=\max _{1 \leq j \leq K_{N}} \frac{h_{\pi(j)} / W}{\lambda_{N}+\mu_{N} g_{\pi(j)}}$. This expression makes it further clear that the jointly optimum spectrum sharing and power control policy maximizing information theoretic throughput capacity of a cognitive uplink under the $K$-out-of- $N$ feedback protocol with average total transmission and interference power constraints is to schedule the SU with the best joint direct and interference channel state among the ones whose interference channel states are fed back to the SBS.

$$
\begin{gathered}
P_{i, N}^{\star}(\boldsymbol{h}, \boldsymbol{g})= \begin{cases}\left(\frac{1}{\lambda_{N}+\mu_{N} g_{i}}-\frac{W}{h_{i}}\right)^{+} & \text {if } i=\arg \max _{1 \leq j \leq N} \frac{h_{j}}{\lambda_{N}+\mu_{N} g_{j}} \\
0 & \text { otherwise. }\end{cases} \\
P_{i, K_{N}}^{\star}(\boldsymbol{h}, \boldsymbol{g})= \begin{cases}\left(\frac{1}{\lambda_{N}+\mu_{N} g_{i}}-\frac{W}{h_{i}}\right)^{+} & \text {if } i=\pi\left(\arg \max _{1 \leq j \leq K_{N}} \frac{h_{\pi(j)}}{\lambda_{N}+\mu_{N} g_{\pi(j)}}\right) \\
0 & \text { otherwise. }\end{cases}
\end{gathered}
$$




\section{IL Networks}

We study the throughput scaling behavior of IL networks under two $\mathrm{CoSs}$ of interest: $\operatorname{CoS}_{\mathrm{IL}}^{\text {Full }}$ and $\operatorname{CoS}_{\mathrm{IL}}^{K}$. In $\mathrm{CoS}_{\mathrm{IL}}^{\text {Full }}$, transmission powers of SUs are limited only by an average total interference power constraint. In this case, the secondary network throughput is given by $R_{\mathrm{IL}}(N)=$ $\mathrm{E}\left[\log \left(X_{N}^{\star}\left(0, \mu_{N}\right)\right) 1_{\left\{X_{N}^{\star}\left(0, \mu_{N}\right) \geq 1\right\}}\right]$. On the other hand, transmission powers of SUs are also limited by the feedback constraint (1d) in $\operatorname{CoS}_{\mathrm{IL}}^{K}$. The secondary network throughput in $\operatorname{CoS}_{\mathrm{IL}}^{K}$ is given by

$$
R_{\mathrm{IL}}\left(K_{N}\right)=\mathrm{E}\left[\log \left(X_{K_{N}}^{\star}\left(0, \mu_{N}\right)\right) 1_{\left\{X_{K_{N}}^{\star}\left(0, \mu_{N}\right) \geq 1\right\}}\right],
$$

where the random variable $X_{K_{N}}^{\star}\left(\lambda_{N}, \mu_{N}\right)$ summarizes the best joint channel state under limited primary cooperation defined as above.

\section{E. IPIL Networks}

Similar to the above cases, we investigate the secondary network throughput scaling behavior of IPIL networks under two CoSs of interest: $\operatorname{CoS}_{\text {IPIL }}^{\text {Full }}$ and $\operatorname{CoS}_{\text {IPIL }}^{K}$. In $\operatorname{CoS}_{\text {IPIL }}^{\text {Full }}$, transmission powers of SUs are limited by individual average transmission power constraints and an average total interference power constraint. Hence, transmission powers of SUs are allocated according to the solution of the following optimization problem:

$$
\begin{aligned}
& \max _{\boldsymbol{P}(\boldsymbol{h}, \boldsymbol{g})} \mathrm{E}_{\boldsymbol{h , g}}\left[\log \left(1+\frac{\boldsymbol{h}^{\top} \boldsymbol{P}(\boldsymbol{h}, \boldsymbol{g})}{W}\right)\right] \\
& \text { subject to : (1b), (1c) and (1e). }
\end{aligned}
$$

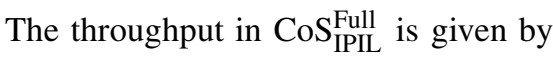

$$
R_{\mathrm{IPIL}}(N)=\mathrm{E}\left[\log \left(X_{N}^{\star}\left(\lambda_{N}, \mu_{N}\right)\right) 1_{\left\{X_{N}^{\star}\left(\lambda_{N}, \mu_{N}\right) \geq 1\right\}}\right],
$$

where $\lambda_{N}$ now represents the Lagrange multiplier associated with individual transmission power constraints. Again, the definition of $X_{N}^{\star}\left(\lambda_{N}, \mu_{N}\right)$ is the same with the one above, except with a change of interpretation of the Lagrange multiplier $\lambda_{N}$ in this network. Hence, although the functional structure of the power control policy is the same for both cases of TPIL and IPIL networks, the resulting transmission powers can be much different. In the first case, $\lambda_{N}$ is chosen to keep the aggregate transmission power around $P_{\text {ave }}$ whenever there is a transmission from the totality of all SUs. Hence, each transmission is expected to occur with power around $P_{\text {ave }}$ in TPIL networks. On the other hand, $\lambda_{N}$ is chosen to keep individual transmission powers around $P_{\text {ave }}$ in IPIL networks. Therefore, considering the spectrum access probability, each transmission is expected to occur with power around $P_{\text {ave }}$ times the probability of being scheduled for transmission in the second case. This difference in turn results in different throughput scaling behavior for both networks as explained in detail in Section IV.

In $\operatorname{CoS}_{\mathrm{IPIL}}^{K}$, in addition to the individual average transmission power and average total interference power constraints, transmission powers of SUs are also limited by the feedback constraint in (1d). Hence, the transmission powers of SUs are allocated according to the solution of the following optimization problem:

$$
\begin{aligned}
& \max _{\boldsymbol{P}(\boldsymbol{h}, \boldsymbol{g})} \mathrm{E}_{\boldsymbol{h , \boldsymbol { g }}}\left[\log \left(1+\frac{\boldsymbol{h}^{\top} \boldsymbol{P}(\boldsymbol{h}, \boldsymbol{g})}{W}\right)\right] \\
& \text { subject to : (1b), (1c), (1d) and (1e). }
\end{aligned}
$$

The throughput in $\operatorname{CoS}_{\text {IPIL }}^{K}$ is given by

$$
R_{\mathrm{IPIL}}\left(K_{N}\right)=\mathrm{E}\left[\log \left(X_{K_{N}}^{\star}\left(\lambda_{N}, \mu_{N}\right)\right) 1_{\left\{X_{K_{N}}^{\star}\left(\lambda_{N}, \mu_{N}\right) \geq 1\right\}}\right] .
$$

Again, the definition of $X_{K_{N}}^{\star}\left(\lambda_{N}, \mu_{N}\right)$ is the same with the one given above, except with a slight change of interpretation of the Lagrange multiplier $\lambda_{N}$ in this network type.

Remark 2: Although the same notations $\lambda_{N}$ and $\mu_{N}$ are used to represent the Lagrange multipliers for different network types, their association to the constraints will be clear from the context. In particular, $\lambda_{N}$ will represent the Lagrange multiplier associated with the total average transmission power constraint in $\operatorname{CoS}_{\mathrm{TPIL}}^{\mathrm{Full}}$ and $\operatorname{CoS}_{\mathrm{TPIL}}^{K}$, whereas it will represent the identical Lagrange multipliers associated with individual average transmission power constraints in $\operatorname{CoS}_{\text {IPIL }}^{\text {Full and }}$ $\mathrm{CoS}_{\text {IPIL }}^{K}$ in the remainder of the paper. Also, $\mu_{N}$ represents the Lagrange multiplier associated with the average total interference power constraint in all CoSs.

Remark 3: In Appendix A, we show that the concentration behavior of the extreme order statistic of an i.i.d. sequence of random variables with a common $\mathrm{CDF} F_{X}$, which does not have to have a closed form expression, is characterized by the functional inverse of the function $G(x)$ characterizing the tail behavior of $F_{X}$, i.e., $\lim _{x \rightarrow \infty} G(x)\left(1-F_{X}(x)\right)=1$. This is the key result used to establish the secondary network throughput scaling behavior under different CoSs.

\section{Analytical Results and Discussions}

In this section, we state the main MDG results under both full and limited cooperation scenarios for different network types. For each network type, we provide the sufficient condition on $K_{N}$ such that the $K$-out-of- $N$ feedback protocol is asymptotically optimal. We also discuss various insights about the derived MDGs. The proofs are relegated to the appendices for the sake of fluency of the paper. We start our discussions by establishing the secondary network throughput scaling when $I$, i.e., the primary's interference power at the SBS, is a fixed but an arbitrary constant. In the next section, we show that the same throughput scaling results continue to hold for randomly varying interference power at the SBS.

\section{A. MDG in IL Networks Under Full and Limited Cooperation Scenarios}

The following theorem establishes the secondary network throughput scaling behavior under $\operatorname{CoS}_{\mathrm{IL}}^{\text {Full }}$ and $\operatorname{CoS}_{\mathrm{IL}}^{K}$.

Theorem 1: Consider an IL network in which the primary's interference power at the SBS is constant. Let $R_{\mathrm{IL}}(N)$ and $R_{\mathrm{IL}}\left(K_{N}\right)$ be the secondary network throughput under 
$\operatorname{CoS}_{\mathrm{IL}}^{\text {Full }}$ and $\operatorname{CoS}_{\mathrm{IL}}^{K}$, respectively. Then, for $1 \leq K_{N} \leq N$, we have

$$
\lim _{N \rightarrow \infty} \frac{R_{\mathrm{IL}}(N)}{\log (N)}=\lim _{N \rightarrow \infty} \frac{R_{\mathrm{IL}}\left(K_{N}\right)}{\log (N)}=\frac{1}{\gamma_{g}} .
$$

Proof: See Appendix B.

Theorem 1 establishes the logarithmic scaling behavior for the secondary network throughput with $N$ under $\operatorname{CoS}_{\mathrm{IL}}^{\text {Full }}$ and $\mathrm{CoS}_{\mathrm{IL}}^{K}$. An important aspect of Theorem 1 is that the derived capacity scaling result under the limited primary cooperation is independent of the scaling behavior of $K_{N}$. Hence, Theorem 1 also indicates that the optimum secondary network throughput scaling behavior in $\operatorname{CoS}_{\mathrm{II}}^{K}$ can be attained even with $K_{N}=O(1)$. This is primarily because the interference channel gains turn out to be the main source of MDGs in IL networks since $\frac{h_{i}}{g_{i}}$ and $\frac{1}{g_{i}}$ have the similar tail behavior (see Appendix B for more details). Thus, the common CDF of the interference channel gains characterizes the asymptotic behavior of $\max _{i} \frac{h_{i}}{g_{i}}$. As a result, scheduling the SU with the smallest interference channel gain for transmission does not change the throughput scaling behavior up to a first order. Direct channel gains only have a second order effect on the achievable throughput levels of the secondary network.

The throughput analysis of fully distributed IL cognitive uplinks in [10] revealed that the secondary network throughput in a fully distributed IL network scales according to $\frac{1}{\mathrm{e} \gamma_{g}} \log (N)$. Here, the factor $\frac{1}{\mathrm{e}}$ represents the throughput loss in the fully distributed mode of operation due to collisions and no transmission events. Thus, the fully distributed IL networks suffer from more than $60 \%$ throughput loss, compared to the IL networks under full or limited primary cooperation scenario, due to uncoordinated transmission strategies of SUs. Note that the fully distributed cognitive uplinks operate with no primary cooperation, i.e., there is no feedback link deployed between the PBS and SBS, and each SU decides whether to transmit or not, only based on the local knowledge of its own direct and interference channel gains. On the other hand, Theorem 1 implies that an IL network with limited primary cooperation under $K$-out-of- $N$ feedback protocol can achieve a much superior throughput performance than that of fully distributed IL networks. Further, perhaps more importantly, this boost in the throughput performance (i.e., almost three times increase) comes only with an $O(1)$ feedback exchange between the PBS and the SBS. This observation suggests that employing the $K$-out-of- $N$ feedback protocol is a much more favorable approach for the primary-secondary feedback problem when compared to the fully distributed implementation of cognitive uplinks.

Furthermore, Theorem 1 reveals that the secondary network throughput scaling under $\operatorname{CoS}_{\mathrm{IL}}^{\mathrm{Full}}$ and $\operatorname{CoS}_{\mathrm{IL}}^{K}$ is controlled by a pre-log factor of $\frac{1}{\gamma_{g}}$ that is determined from the behavior of the CDF of the interference channel gains around zero. The pre-log factor is equal to $\frac{2}{c}, \frac{1}{m}$ and 1 for the Weibull- $c$, Nakagami- $m$ and Rician- $k$ distributed interference channel gains, respectively. The effect of $\gamma_{g}$ on $R_{\mathrm{IL}}(N)$ and $R_{\mathrm{IL}}\left(K_{N}\right)$ has an engineering interpretation. For a given fading model for interference channels, $\gamma_{g}$ is a measure for the proximity of the interference channel power gains to zero. That is, small values of $\gamma_{g}$ imply that the interference channel gains take values close to zero with high probability, and vice versa. Thus, as $\gamma_{g}$ increases, the interference channel gains become large, and consequently SUs reduce their transmission powers in order to meet the average interference power constraint at the PBS. As a result, the secondary network throughput decreases as $\gamma_{g}$ becomes large.

At this point, it is also insightful to compare the result of Theorem 1 with the capacity scaling behavior under the following simple heuristic approach. Since there is no limitation on the transmission power, one may speculate at the first sight that the stated throughput scaling behavior in the IL networks can be easily obtained by scheduling a SU with transmission power equal to $N P_{\text {ave }}$, which will lead to throughput scaling as $\log (N)$. For $\gamma_{g}<1$, the proposed heuristic cannot achieve full MDG in IL networks since Theorem 1 shows that the throughput in IL networks under optimal power allocation policy is superior to $\log (N)$ and scales according to $\frac{1}{\gamma_{g}} \log (N)$. For $\gamma_{g}>1$, on the other hand, it can be shown that this heuristic power allocation policy violates the average interference constraint as $N$ becomes large, i.e., transmission power grows at a rate larger than the decay in the minimum interference channel gain. Therefore, apart from the the peculiar case $\gamma_{g}=1$, which holds for the Rayleigh fading scenario, the optimum power control policy must be followed to have a complete capacity scaling characterization for the IL networks.

In Appendix B, we show that the Lagrange multipliers $\mu_{N}$ converge to $\frac{1}{Q_{\text {ave }}}$ in $\operatorname{CoS}_{\mathrm{IL}}^{\text {Full }}$. This finding can be used to study the effect of $Q_{\text {ave }}$ on the secondary network throughput under $\operatorname{CoS}_{\mathrm{IL}}^{\text {Full }}$. Based on our analysis in Appendix B, we characterize the second order effects of $Q_{\text {ave }}$ and fading parameters on the secondary network throughput under $\operatorname{CoS}_{\mathrm{IL}}^{\mathrm{Full}}$ for finite numbers of SUs by bounding $R_{\mathrm{IL}}(N)$ from below and above as

$$
\begin{aligned}
(1-\epsilon) & \frac{1}{\gamma_{g}} \log (N)+\log \left(Q_{\text {ave }}\right)+\frac{1}{\gamma_{g}} \log \left(\eta_{g} \mathrm{E}\left[h^{\gamma_{g}}\right]\right)+O \\
\leq & R_{\mathrm{IL}}(N) \leq(1+\epsilon) \frac{1}{\gamma_{g}} \log (N)+\log \left(Q_{\text {ave }}\right) \\
& +\frac{1}{\gamma_{g}} \log \left(\eta_{g} \mathrm{E}\left[h^{\gamma_{g}}\right]\right)+O
\end{aligned}
$$

for all $\epsilon>0$ and $N$ large enough (i.e., see (9)). Hence, an increase in $Q_{\text {ave }}$ leads to a logarithmic increase in $R_{\mathrm{IL}}(N)$, implying that $Q_{\text {ave }}$ has a logarithmic effect on $R_{\mathrm{IL}}(N)$. Furthermore, the second order effects of the direct and interference channel fading models on $R_{\mathrm{IL}}(N)$ can be thought to be embodied in $\frac{1}{\gamma_{g}} \log \left(\eta_{g} \mathrm{E}\left[h^{\gamma_{g}}\right]\right)$, where $h$ is a generic nonnegative random variable having the same CDF with direct channel gains.

Remark 4: Operating in the IL scenario does not necessarily imply that the average transmission powers of SUs are infinite. It is easy to show that when the distribution of interference channel gains belongs to class- $C$ distributions with $\gamma_{g}>1$, the average transmission powers of SUs are finite. 


\section{B. MDG in IPIL Networks Under Full and Limited Cooperation Scenarios}

Now, we investigate throughput scaling laws in IPIL networks under full and limited cooperation scenarios. The next theorem establishes the secondary network throughput scaling behavior under $\operatorname{CoS}_{\text {IPIL }}^{\text {Full }}$ and $\operatorname{CoS}_{\text {IPIL }}^{K}$.

Theorem 2: Consider an IPIL network in which the primary's interference power at the SBS is constant. Let $R_{\mathrm{IPIL}}(N)$ and $R_{\mathrm{IPIL}}\left(K_{N}\right)$ be the secondary network throughput in this network under $\operatorname{CoS}_{\text {IPIL }}^{\text {Full }}$ and $\operatorname{CoS}_{\text {IPIL }}^{K}$, respectively, for $1 \leq K_{N} \leq N$. Then,

$$
\lim _{N \rightarrow \infty} \frac{R_{\text {IPIL }}\left(K_{N}\right)}{\log (N)}=\lim _{N \rightarrow \infty} \frac{R_{\text {IPIL }}(N)}{\log (N)}=\min \left(1, \frac{1}{\gamma_{g}}\right) .
$$

Proof: See [27].

Theorem 2 establishes the logarithmic scaling behavior of the secondary network throughput under $\operatorname{CoS}_{\text {IPIL }}^{\text {Full and }} \operatorname{CoS}_{\text {IPIL }}^{K}$ as a function of the number of SUs. For $\operatorname{CoS}_{\mathrm{IPIL}}^{K}$, the scaling behavior does not depend on the number of interference channel gains available at the SBS. Hence, even for $K_{N}=O(1)$, a secondary network under $\operatorname{CoS}_{\text {IPIL }}^{K}$ can achieve the same scaling behavior as the one achieved under $\mathrm{CoS}_{\text {IPIL }}^{\text {Full }}$, which implies a tremendous reduction in the primary-secondary feedback load. We note that such a behavior was also observed for the IL networks.

Theorem 2 also reveals the effect of parameters of the interference fading model on the scaling behavior of $R_{\text {IPIL }}(N)$ and $R_{\text {IPIL }}\left(K_{N}\right)$, which appears as the pre-log factor of $\min \left(1, \frac{1}{\gamma_{g}}\right)$. This effect has the following interpretation. For $\gamma_{g}<1$, random interference channel gains take values close to zero with high probability. As a result, the average interference power constraint becomes increasingly looser, and the transmission powers of SUs become mainly limited by the individual average transmission power constraints, i.e., secondary network behaves as a primary uplink with individual power constraints only. The throughput of a primary uplink with individual power constraints scales according to $\log N$ (i.e., see [27]). Hence, the secondary network throughput scales according to $\log (N)$ for $\gamma_{g}<1$. For $\gamma_{g}>1$, random interference channel gains take large values away from zero with high probability, when compared with the case of $\gamma_{g}<1$. Thus, the average interference power constraint becomes more stringent, and the secondary network behaves as an IL network. This leads to the result that the secondary network throughput scales according to $\frac{1}{\gamma_{g}} \log (N)$ for $\gamma_{g}>1$.

From a more heuristic perspective, the effect of the pre$\log$ factor $\min \left(1, \frac{1}{\gamma_{g}}\right)$ has the following interpretation. By removing the interference power constraint from an IPIL network, we obtain a primary uplink with individual power constraints whose throughput, which we represent as $R_{\mathrm{IPL}}(N)$, can be shown to scale according to $\log (N)$. On the other hand, by removing the individual power constraints from an IPIL network, we obtain an IL network whose throughput $R_{\mathrm{IL}}(N)$ can be shown to scale according to $\frac{1}{\gamma_{g}} \log (N)$. Thus, $R_{\text {IPIL }}(N)$ is upper bounded by the communication rates obtained in both cases. Depending on the value of $\gamma_{g}$, one of the upper bounds bites. That is, for $\gamma_{g}<1, R_{\mathrm{IPL}}(N)$ bound is tighter than $R_{\mathrm{IL}}(N)$, and as a result the secondary network throughput scales according to $\log (N)$. For $\gamma_{g}>1, R_{\mathrm{IL}}(N)$ bound is tighter than $R_{\mathrm{IPL}}(N)$, and the secondary network throughput scales according to $\frac{1}{\gamma_{g}} \log (N)$. It should be noted these arguments can only provide us with an upper bound. More analysis is needed to establish the lower bounds with the same scaling behavior as well as for deriving the scaling behavior of $R_{\mathrm{IPIL}}\left(K_{N}\right)$, i.e., see [27].

\section{MDG in TPIL Networks Under Full and Limited Cooperation Scenarios}

Finally, we discuss the throughput scaling behavior of TPIL networks under full and limited cooperation scenarios. Some parts of our results for TPIL networks have been partially appeared in [26]. Here, we mention them for the sake of completeness of the paper. We also provide additional important observations and insights regrading the observed MDGs in TPIL networks that did not appear in [26]. The next theorem establishes the throughput scaling behavior for $R_{\text {TPIL }}(N)$ and $R_{\mathrm{TPIL}}\left(K_{N}\right)$ as a function of the number of SUs.

Theorem 3: Consider a TPIL network in which the primary's interference power at the SBS is constant. Let $K_{N}$ grow to infinity at a rate $K_{N}=o(N)$ and let $R_{\text {TPIL }}(N)$ and $R_{\text {TPIL }}\left(K_{N}\right)$ be the secondary network throughput in this network under $\operatorname{CoS}_{\mathrm{TPIL}}^{\mathrm{Full}}$ and $\operatorname{CoS}_{\mathrm{TPIL}}^{K}$, respectively. Then

$$
\lim _{N \rightarrow \infty} \frac{R_{\mathrm{TPIL}}\left(K_{N}\right)}{\log \left(\log \left(K_{N}\right)\right)}=\lim _{N \rightarrow \infty} \frac{R_{\mathrm{TPIL}}(N)}{\log (\log (N))}=\frac{1}{n_{h}} .
$$

Proof: See [26].

Theorem 3 indicates that the secondary network throughput scales double logarithmically under $\operatorname{CoS}_{\mathrm{TPIL}}^{\text {Full }}$ and $\operatorname{CoS}_{\mathrm{TPIL}}^{K}$ with $N$ and $K_{N}$, respectively. Hence, for $K_{N}=N^{\delta}$ and $\delta \in(0,1)$, the secondary network throughput scaling behavior under $\operatorname{CoS}_{\mathrm{TPIL}}^{\mathrm{Full}}$ and $\operatorname{CoS}_{\mathrm{TPIL}}^{K}$ are the same. Since $\delta$ can be chosen arbitrarily close to zero, this result implies that the amount of feedback in the feedback link can be dramatically reduced while the secondary network still achieves the same scaling behavior as the one achieved with the full primary cooperation.

At this point, it is again important to compare and contrast this result with the throughput scaling behavior obtained in [10] for the fully distributed mode of operation. It was shown in [10] that the secondary network throughput in fully distributed TPIL networks scales according to $\frac{1}{\mathrm{e} n_{h}} \log \log (N)$, which exhibits a drastic reduction in rate when compared to the throughput scaling behavior of TPIL networks under full or limited primary cooperation scenario. In particular, Theorem 3 shows that a TPIL network with limited primary cooperation under $K$-out-of- $N$ feedback protocol, with $N^{\delta}$ and $\delta(0,1)$, is capable of achieving the same MDG as that of the TPIL networks under full primary's cooperation. Further, the feedback load can be reduced significantly by taking $\delta$ a small positive number at the expense of rate loss effects to a second order. From an engineering point of view, a prime design implication of this result is that we can boost the secondary network communication rates almost three times (when compared to the fully distributed mode of operation) 
if a low-rate feedback link can be established between the PBS and SBS.

The dependence of MDGs in $\operatorname{CoS}_{\mathrm{TPIL}}^{K}$ on $K_{N}$ indicates that the direct channel gains are the major source of MDGs in TPIL networks. This is mainly because the Lagrange multipliers $\lambda_{N}$ cannot be made arbitrarily close to zero in this case, and as a result, the asymptotic behavior of $\max _{1 \leq i \leq K_{N}} \frac{h_{\pi(i)}}{\lambda_{N}+\mu_{N} g_{\pi(i)}}$ is primarily governed by the distribution of direct channel power gains. Larger $K_{N}$ implies that more interference channel gains are available at the SBS, and we observe a corresponding increase in the MDG.

Theorem 3 also reveals that the secondary network throughput scaling behavior under $\operatorname{CoS}_{\mathrm{TPIL}}^{\mathrm{Full}}$ and $\mathrm{CoS}_{\mathrm{TPIL}}^{K}$ is controlled by a pre-log factor of $\frac{1}{n_{h}}$. To put it another way, the available degrees of freedom for the cognitive multiple access channel in question reversely depends on the tail decay rate of the CDF of the direct channel power gains. The pre-log factor is equal to $\frac{2}{c}$ for the Weibull- $c$ distributed direct channel gains, and equal to 1 for Rayleigh, Rician- $k$ and Nakagami- $m$ distributed direct channel gains.

One can show that the Lagrange multipliers $\lambda_{N}$ converge to $\frac{1}{P_{\text {ave }}}$ as $N$ becomes large in both cases of $\operatorname{CoS}_{\mathrm{TPIL}}^{\mathrm{Full}}$ and $\operatorname{CoS}_{\mathrm{TPIL}}^{K}$ (i.e., see [27]). This finding is helpful to study the second order effects of the average total transmission power constraint $P_{\text {ave }}$ on the secondary network throughput under $\operatorname{CoS}_{\mathrm{TPIL}}^{\text {Full }}$ and $\mathrm{CoS}_{\text {TPIL }}^{K}$. That is, $R_{\text {TPIL }}(N)$ can be bounded from above and below as

$$
\begin{aligned}
(1 & -\epsilon) \frac{1}{n_{h}} \log \log (N)+\log \left(P_{\mathrm{ave}}\right)+\frac{1}{n_{h}} \log \left(\frac{1}{\beta_{h}}\right)+O(1) \\
& \leq R_{\mathrm{TPIL}}(N) \\
& \leq(1+\epsilon) \frac{1}{n_{h}} \log \log (N)+\log \left(P_{\mathrm{ave}}\right)+\frac{1}{n_{h}} \log \left(\frac{1}{\beta_{h}}\right)+O(1),
\end{aligned}
$$

for all $\epsilon>0$ and $N$ large enough (i.e., see [27]). Therefore, an increase in $P_{\text {ave }}$ results in a corresponding logarithmic increase in $R_{\text {TPIL }}(N)$, implying that $P_{\text {ave }}$ has a logarithmic effect on $R_{\text {TPIL }}(N)$. For a given fading model for direct channel gains, the constant term $\frac{1}{n_{h}} \log \left(\frac{1}{\beta_{h}}\right)$ above can be thought of being the second order effect of the fading model on $R_{\text {TPIL }}(N)$ for finitely many SUs. Similar bounds also apply to $R_{\text {TPIL }}\left(K_{N}\right)$. The $\frac{1}{n_{h}} \log \left(\frac{1}{\beta_{h}}\right)$ term is equal to $\log \left(\frac{1}{k+1}\right)$ for the Rician- $k$ distributed direct channel gains, and equal to $\log \left(\frac{1}{m}\right)$ for the Nakagami- $m$ distributed direct channel gains. This implies that for a fixed number of SUs, as the Rician factor $k$ or the Nakagami parameter $m$ increases, we observe a logarithmic reduction in the secondary network throughput. The reason for this behavior is that direct channel gains become more deterministic as $k$ or $m$ increases, and as a result, the MDG drops since it depends on the dynamic range of the CDF of the direct channel gains [25].

For the Weibull- $c$ distributed direct channel gains, the $\frac{1}{n_{h}} \log \left(\frac{1}{\beta_{h}}\right)$ term is equal to $\log \left(\frac{1}{\Gamma\left(1+\frac{2}{c}\right)}\right)$, which first increases and then decreases as the Weibull fading parameter $c$ grows large. Here, $\Gamma(\cdot)$ represents the gamma function. This behavior can be explained as follows. For small values of $c$, the Weibull-c distribution is concentrated around zero, i.e., it is almost deterministic. On the other hand, its dynamic range expands as $c$ increases. Thus, the second order term $\log \left(\frac{1}{\Gamma\left(1+\frac{2}{c}\right)}\right)$ in the throughput expression increases as $c$ increases from zero. However, as $c$ becomes large, after a certain point, the Weibull distribution starts to concentrate around one, i.e., it becomes deterministic again, and as a result the second order term $\log \left(\frac{1}{\Gamma\left(1+\frac{2}{c}\right)}\right)$ drops again. Finally, we note that $P_{\text {ave }}$ and the direct channel fading distribution parameters have the similar logarithmic second order effects on the secondary network throughput under $\operatorname{CoS}_{\mathrm{TPIL}}^{K}$ (i.e., see [27]).

Remark 5: To prove Theorem 3, we show that the throughput of a primary multiple access network with a total power constraint $R_{\mathrm{TPL}}(N)$ scales according to $\lim _{N \rightarrow \infty} \frac{R_{\mathrm{TPL}}(N)}{\log \log (N)}=$ $\frac{1}{n_{h}}$. This result is used to establish the upper bound on the secondary network throughput under $\operatorname{CoS}_{\mathrm{TPIL}}^{\mathrm{Full}}$. (i.e., see [27] for more details.)

Our next theorem establishes an important convergence behavior for the total interference power at the PBS under $\mathrm{CoS}_{\mathrm{TPIL}}^{K}$ as the number of SUs grows large.

Theorem 4: Let $I_{K_{N}}$ be the secondary network interference power at the PBS under $\operatorname{CoS}_{\mathrm{TPIL}}^{K}$. For $K_{N}=o(N)$, $\lim _{N \rightarrow \infty} I_{K_{N}}=0$ almost surely and $\lim _{N \rightarrow \infty} \mathrm{E}\left[I_{K_{N}}\right]=0$.

Proof: See [26].

This convergence result can be justified by the fact that $g_{K_{N}: N}$, i.e., the largest interference channel gain available at the SBS under the $K$-out-of- $N$ feedback protocol, converges to zero as $N$ becomes large for $K_{N}=o(N)$. An important practical consequence of Theorems 3 and 4 is that for $K_{N}=$ $N^{\delta}$ and $\delta \in(0,1)$, the secondary network under $\operatorname{CoS} S_{\text {TPIL }}^{K}$ achieves the optimum throughput scaling behavior while the interference at the PBS becomes negligible as $N$ grows large. This finding implies that the secondary network can co-exist with the primary network by virtually causing no interference, and yet still achieving the optimum data rates.

It is also important to note that Theorem 4 implies the existence of a constant $N^{\prime}$ such that for all $N \geq N^{\prime}$, the average interference power constraint at the PBS cannot be satisfied with equality. Therefore, the Lagrange multipliers associated with the average interference power constraint become zero for all $N$ large enough, i.e., $\mu_{N}=0$ for all $N \geq N^{\prime}$. As a result, the SBS just requires the index set $I_{K_{N}}=\left\{i: i=\pi(j), 1 \leq j \leq K_{N}\right\}$ to choose the SU with the best direct channel gain for optimum power allocation. Thus, the PBS only needs to transmit $K_{N}\left\lceil\log _{2}(N)\right\rceil$ bits of feedback to the SBS. From a practical point of view, this phenomenon provides an extra reduction in the total feedback load required to achieve the optimum throughput scaling for cognitive radio networks.

\section{EXTENSIONS TO RANDOMLY VARYING INTERFERENCE POWER AT THE SBS}

In this section, we show that the throughput scaling results in Theorems 1-3 continue to hold even when the primary's 
interference power at the SBS, i.e., I, changes randomly between fading blocks. To this end, we assume $I$ is a positive random variable with a finite mean. Next theorem establishes the secondary network throughput scaling in IL networks with randomly varying interference power at the SBS.

Theorem 5: Consider an IL network wherein the primary's interference power at the SBS varies randomly between fading blocks, and has a finite mean. Let $R_{\mathrm{IL}}(N)$ and $R_{\mathrm{IL}}\left(K_{N}\right)$ be the secondary network throughput under $\operatorname{CoS}_{\mathrm{IL}}^{\text {Full }}$ and $\operatorname{CoS}_{\mathrm{IL}}^{K}$, respectively, in such a network. Then, for $1 \leq K_{N} \leq N$, we have

$$
\lim _{N \rightarrow \infty} \frac{R_{\mathrm{IL}}(N)}{\log (N)}=\lim _{N \rightarrow \infty} \frac{R_{\mathrm{IL}}\left(K_{N}\right)}{\log (N)}=\frac{1}{\gamma_{g}} .
$$

Proof: We first note that $R_{\mathrm{IL}}\left(K_{N}\right) \leq R_{\mathrm{IL}}(N)$ as the feedback limitations reduces the secondary network throughput. Here, we establish an upper bound on $R_{\mathrm{IL}}(N)$ as follows. Let $\bar{R}(N)$ be the sum-rate of the same network when $I=0$. We have $R_{\mathrm{IL}}(N) \leq \bar{R}(N)$ since the secondary network does not receive any interference from the primary network in this case, and thus can achieve a higher sum-rate. Further, under the assumption of $I=0$, the total background noise plus the primary interference power at the SBS is constant, and therefore, Theorem 1 above can be used to derive the scaling behavior of $\bar{R}(N)$. The scaling behavior of $\bar{R}(N)$ is given by $\lim _{N \rightarrow \infty} \frac{\bar{R}(N)}{\log (N)}=\frac{1}{\gamma_{g}}$ according to Theorem 1 . Thus, we have $\lim \sup _{N \rightarrow \infty} \frac{R_{\mathrm{LL}}\left(K_{N}\right)}{\log (N)} \leq \lim \sup _{N \rightarrow \infty} \frac{R_{\mathrm{LL}}(N)}{\log (N)} \leq$ $\lim _{N \rightarrow \infty} \frac{\bar{R}(N)}{\log (N)}=\frac{1}{\gamma_{g}}$. To prove the other direction, we use the same techniques introduced in Appendix B. Consider an IL network under $\operatorname{CoS}_{\mathrm{IL}}^{K}$ with the suboptimal power control policy $\hat{P}_{i, N}^{\mathrm{IL}}(\mathrm{g})=\frac{Q_{\mathrm{ave}}}{g_{i}} 1_{\left\{g_{i}=\min _{1 \leq j \leq K_{N}} g_{j}\right\}}$. Following the same steps in Appendix B, it can be easily shown that $\hat{P}_{i, N}^{\mathrm{IL}}(\mathrm{g})$ is a feasible power control policy when $N$ is large enough. Thus, for $N$ large enough we have,

$$
\begin{aligned}
R_{\mathrm{IL}}\left(K_{N}\right) \geq & \log \left(Q_{\text {ave }}\right)+\mathrm{E}\left[\log \left(\frac{h}{N_{0}+I}\right)\right] \\
& +\mathrm{E}\left[\log \left(\frac{1}{g_{\min }(N)}\right)\right], \\
& \stackrel{(a)}{\geq} \log \left(Q_{\text {ave }}\right)+\mathrm{E}[\log (h)]-\log \left(N_{0}+\mathrm{E}[I]\right) \\
& +\mathrm{E}\left[\log \left(\frac{1}{g_{\min }(N)}\right)\right],
\end{aligned}
$$

where $h$ is a generic random variable distributed according to $F_{h}, g_{\min }(N)=\min _{1 \leq i \leq K_{N}} g_{i}$ and $(a)$ follows from Jensen's inequality. Since, $\lim _{N \rightarrow \infty} \frac{E\left[\log \left(\frac{1}{g \min (N)}\right)\right]}{\log (N)}=\frac{1}{\gamma_{g}}$ (see Appendix B for more details), we have $\liminf _{N \rightarrow \infty} \frac{R_{\mathrm{LL}}(N)}{\log (N)} \geq \liminf _{N \rightarrow \infty} \frac{R_{\mathrm{LL}}\left(K_{N}\right)}{\log (N)} \geq \frac{1}{\gamma_{g}}$, which completes the proof.

Theorem 5 shows that the secondary network throughput under $\operatorname{CoS}_{\mathrm{IL}}^{\mathrm{Full}}$ and $\operatorname{CoS}_{\mathrm{IL}}^{K}$ scales according to $\frac{1}{\gamma_{g}} \log (N)$ even when $I$ varies randomly. Moreover, Theorem 5 implies that the $K$-out-of- $N$ feedback protocol with $K_{N}=O(1)$ is asymptotically optimum for IL networks with randomly varying interference power at the SBS. This extension formally reveals that the capacity scaling laws in Theorem 1 are still valid in more realistic communication scenarios wherein the primary's interference power at the SBS varies randomly between fading blocks. The next theorem establishes the capacity scaling behavior of IPIL networks under randomly varying interference power at the SBS.

Theorem 6: Consider an IPIL network wherein the primary's interference power at the SBS varies randomly between fading blocks, and has a finite mean. Let $R_{\mathrm{IPIL}}(N)$ and $R_{\text {IPIL }}\left(K_{N}\right)$ be the secondary network throughput under $\operatorname{CoS}_{\text {IPIL }}^{\text {Full }}$ and $\operatorname{CoS}_{\text {IPIL }}^{K}$, respectively. Then, for $1 \leq K_{N} \leq N$, we have

$$
\lim _{N \rightarrow \infty} \frac{R_{\mathrm{IPIL}}(N)}{\log (N)}=\lim _{N \rightarrow \infty} \frac{R_{\mathrm{IPIL}}\left(K_{N}\right)}{\log (N)}=\min \left(1, \frac{1}{\gamma_{g}}\right) .
$$

Proof: The proof follows from steps similar to those in the proof of Theorem 5, and is skipped to avoid repetition.

According to Theorem 6, the secondary network throughput in IPIL networks with randomly varying interference power at the SBS scales according to $\min \left(1, \frac{1}{\gamma_{g}}\right) \log (N)$ under both

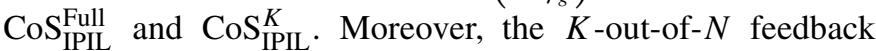
protocol for $K_{N}=O$ (1) is asymptotically optimum for IPIL networks when $I$ is random. This observation implies that the capacity scaling results in Theorem 2 continue to hold for IPIL networks wherein the primary's interference power at the SBS is a random variable with finite mean. The next theorem provides the similar extension for the TPIL networks with randomly varying primary interference power.

Theorem 7: Consider a TPIL network in which the primary's interference power at the SBS changes randomly between fading blocks, and has a finite mean. Let $K_{N}$ grow to infinity at a rate $K_{N}=o(N)$ and let $R_{\text {TPIL }}(N)$ and $R_{\text {TPIL }}\left(K_{N}\right)$ be the secondary network throughput in this network under $\operatorname{CoS}_{\mathrm{TPIL}}^{\mathrm{Full}}$ and $\operatorname{CoS}_{\mathrm{TPIL}}^{K}$, respectively. Then, we have

$$
\lim _{N \rightarrow \infty} \frac{R_{\mathrm{TPIL}}\left(K_{N}\right)}{\log \left(\log \left(K_{N}\right)\right)}=\lim _{N \rightarrow \infty} \frac{R_{\mathrm{TPIL}}(N)}{\log (\log (N))}=\frac{1}{n_{h}} .
$$

Proof: The proof follows from steps similar to those in the proof of Theorem 5, and is skipped to avoid repetition.

\section{NumeriCAl RESUlts}

In this section, we numerically evaluate the throughput scaling behavior of secondary networks under different CoSs with full and limited primary's cooperation. In Figs. $2-4$, it is assumed that primary interference power at the SBS is a constant such that the noise plus interference power at the SBS, which is $N_{0}+I$, is normalized to one. Figure 5 studies the secondary network throughput scaling behavior when the primary's interference power is modelled as an exponentially distributed random variable. Due to space limitations, we refer interested readers to the technical report [27] for a more extensive numerical study of the secondary network throughput scaling laws under $\operatorname{CoS}_{\mathrm{TPIL}}^{\mathrm{Full}}$ and $\operatorname{CoS}_{\mathrm{TPIL}}^{K}$, as well as convergence behavior of interference power at the PBS under $\operatorname{CoS}_{\mathrm{TPIL}}^{K}$. We start by presenting our numerical results for IL networks under full and limited cooperation scenarios.

We plot the secondary network throughput under $\operatorname{CoS}_{\mathrm{IL}}^{\text {Full }}$ and $\mathrm{CoS}_{\mathrm{IL}}^{K}$ as a function of the number of SUs for different communication environments in Figs. 2(a)-(c). In these 


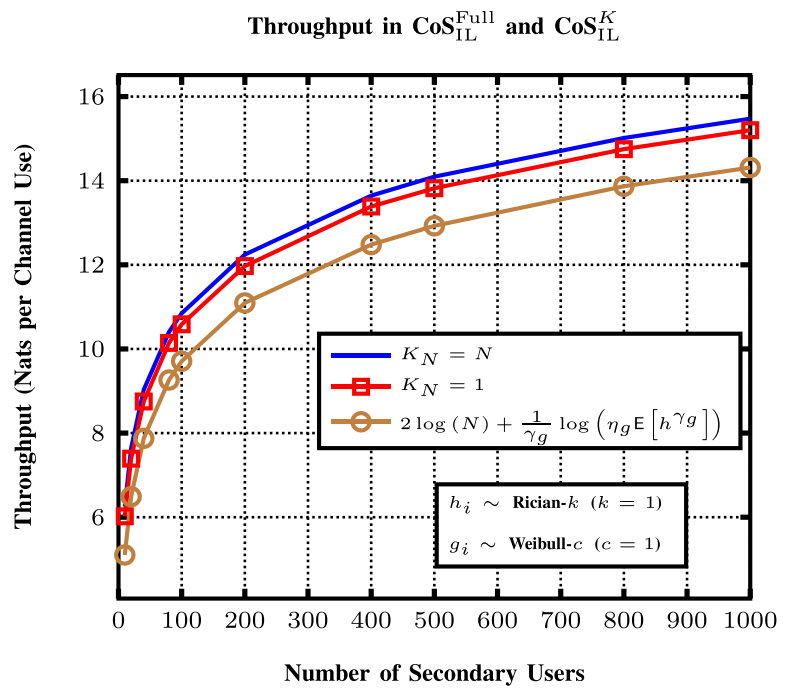

(a)

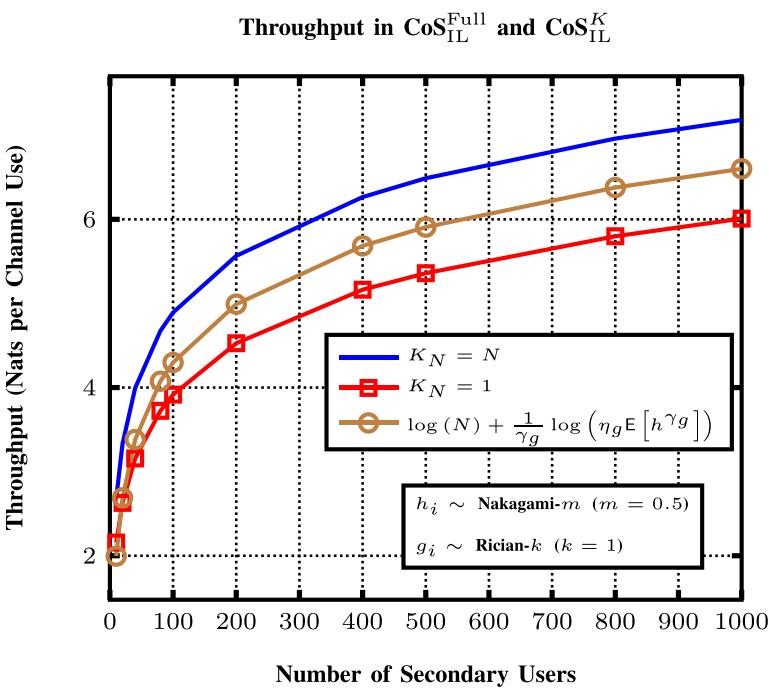

(c)

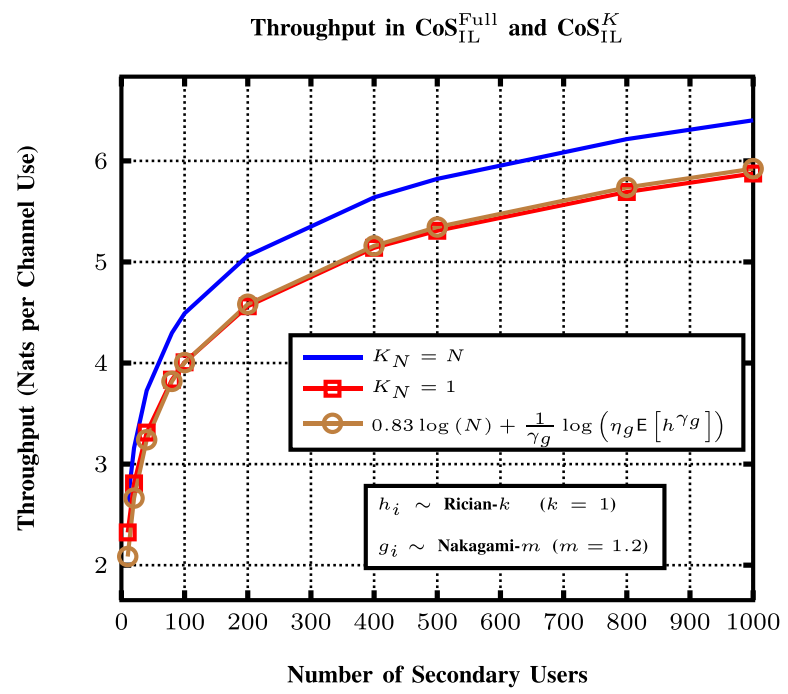

(b)

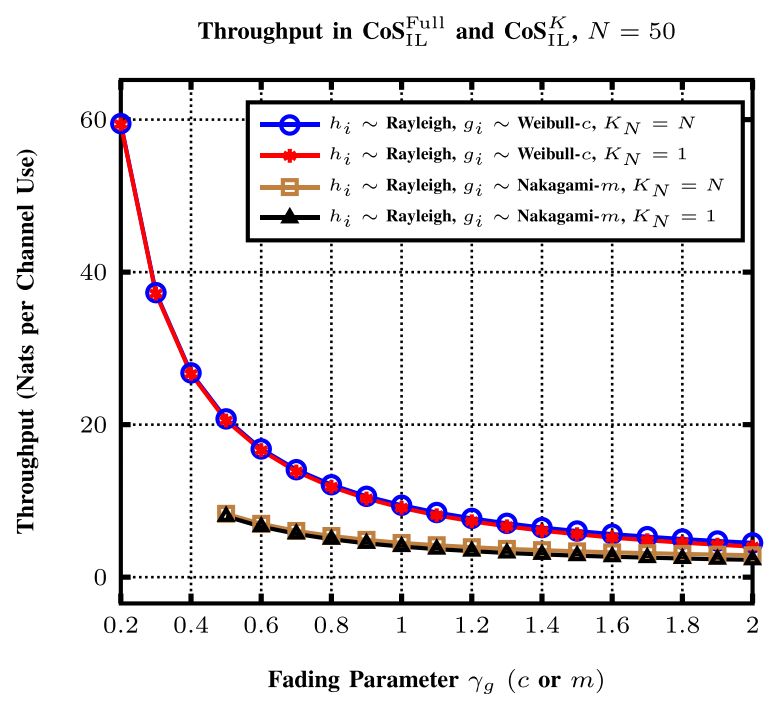

(d)

Fig. 2. Secondary network throughput scaling under $\operatorname{CoS}_{\mathrm{IL}}^{\mathrm{Full}}\left(K_{N}=N\right)$ and $\operatorname{CoS}_{\mathrm{IL}}^{K}\left(K_{N}=1\right)$ for different communication environments (a)-(c). Secondary network throughput under $\operatorname{CoS}_{\mathrm{IL}}^{\text {Full }}$ and $\operatorname{CoS}_{\mathrm{IL}}^{K}$ as a function of $\gamma_{g}$ for $N=50$ for different communication environments (d). $Q_{\text {ave }}$ is set to $0 \mathrm{~dB}$.

figures, the curves with $K_{N}=N$ represent the secondary network throughput under $\operatorname{CoS}_{\mathrm{IL}}^{\text {Full }}$, and the curves with $K_{N}=1$ represent the secondary network throughput under $\operatorname{CoS}_{\mathrm{IL}}^{K}$. For $K_{N}=1$, the PBS only feeds back the smallest interference channel gain to the SBS. $Q_{\text {ave }}$ is set to $0 \mathrm{~dB}$. Similar qualitative behavior continues to hold for other values of $Q_{\text {ave }}$. In Fig. 2(a), direct channel gains are distributed according to the Rician- $k$ fading model with $k=1$, and interference channel gains are distributed according to the Weibull- $c$ fading model with $c=1$. Figure 2 shows that the secondary network throughput scales according to $\frac{2}{c} \log (N)$ when interference channel gains are Weibull- $c$ distributed; a behavior which was predicted by Theorem 1 .

In Fig. 2(b), direct channel gains are distributed according to the Rician- $k$ fading model with $k=1$, and interference channel gains are distributed according to the Nakagami- $m$ fading model with $m=1.2$. Figure 2(b) reveals that the secondary network throughput scales according to $\frac{1}{m} \log (N)$ when interference channel gains are Nakagami- $m$ distributed, which is in accordance with Theorem 1. In Fig. 2(c), direct channel gains are distributed according to the Nakagami- $m$ fading model with $m=0.5$, and interference channel gains are distributed according to the Rician- $k$ fading model with $k=1$. As Fig. 2(c) shows, the secondary network throughput scales according to $\log (N)$ when interference channel gains are distributed according to the Rician- $k$ fading model, which is also in harmony with Theorem 1. Figures 2(a)-(c) also demonstrate that the throughput loss due to implementing the $K$-out-of- $N$ feedback protocol is within one nat per channel use when compared to the full primary cooperation scenario. This observation signifies that the $K$-out-of- $N$ feedback protocol is an effective primary-secondary feedback reduction policy for interference limited cognitive radio networks even for finitely many SUs. 


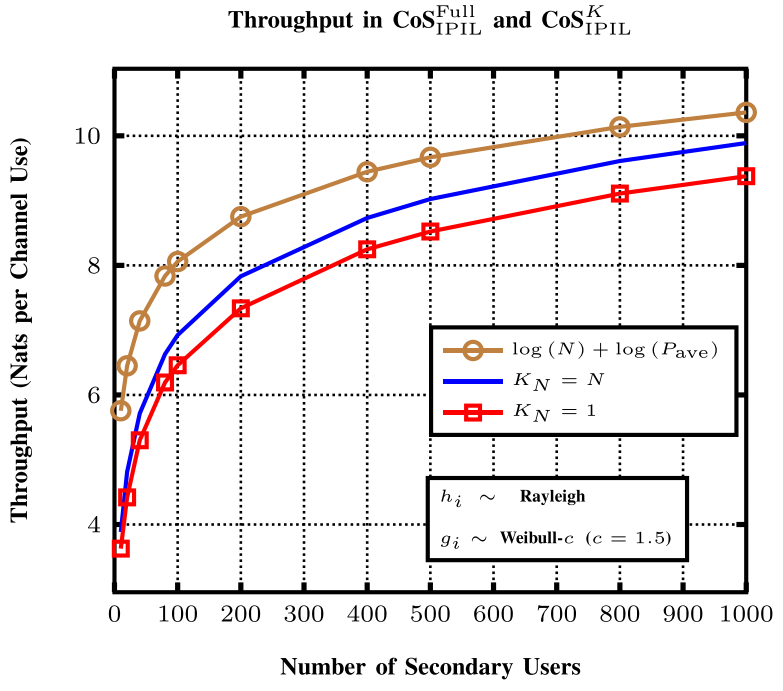

(a)

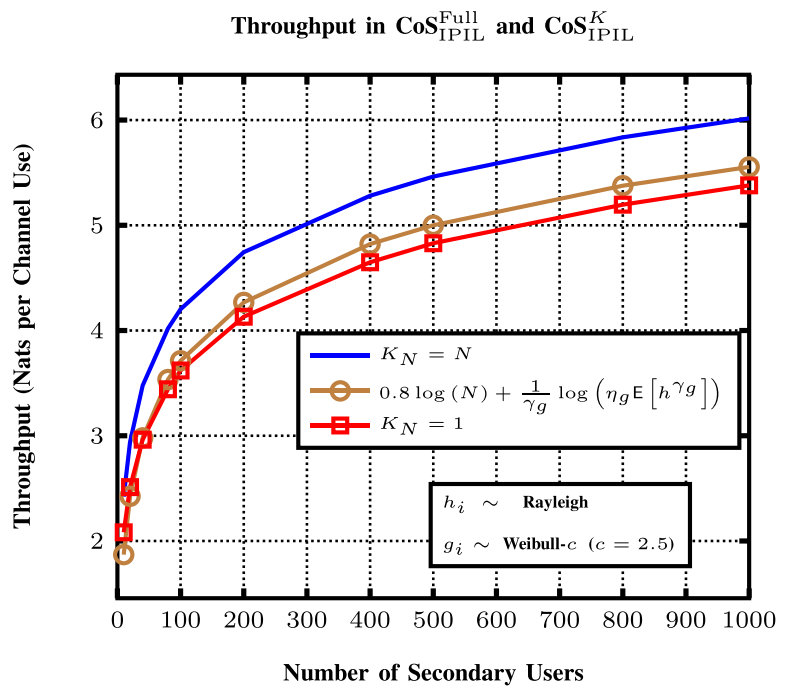

(b)

Fig. 3. Secondary network throughput scaling under $\operatorname{CoS}_{\mathrm{IPIL}}^{\mathrm{Full}}\left(K_{N}=N\right)$ and $\operatorname{CoS}_{\mathrm{IPIL}}^{K}\left(K_{N}=1\right)$ for different communication environments. $P_{\text {ave }}$ and $Q_{\mathrm{ave}}$ are set to $15 \mathrm{~dB}$ and $0 \mathrm{~dB}$, respectively.

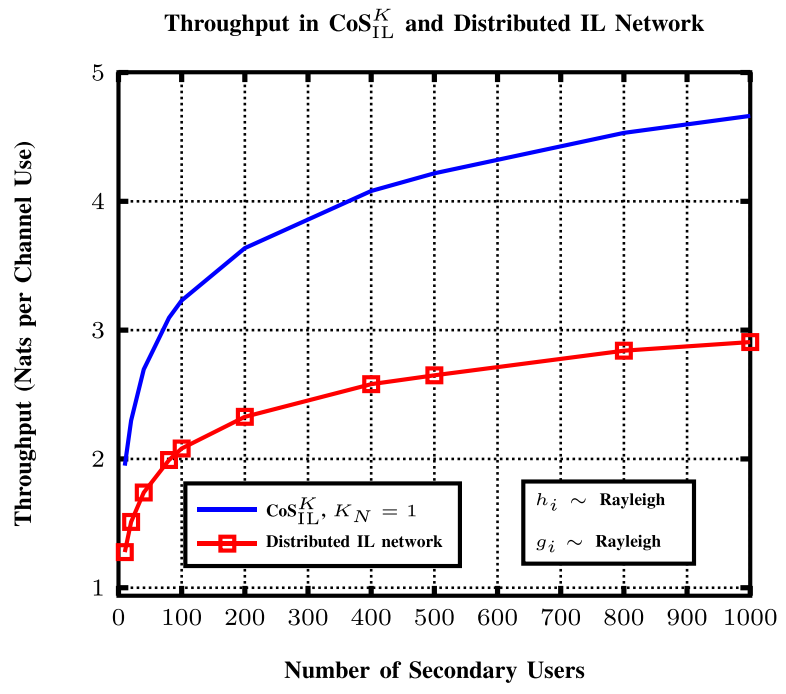

(a)
Throughput in $\operatorname{CoS}_{\mathrm{IPIL}}^{K}$ and Distributed IPIL Network

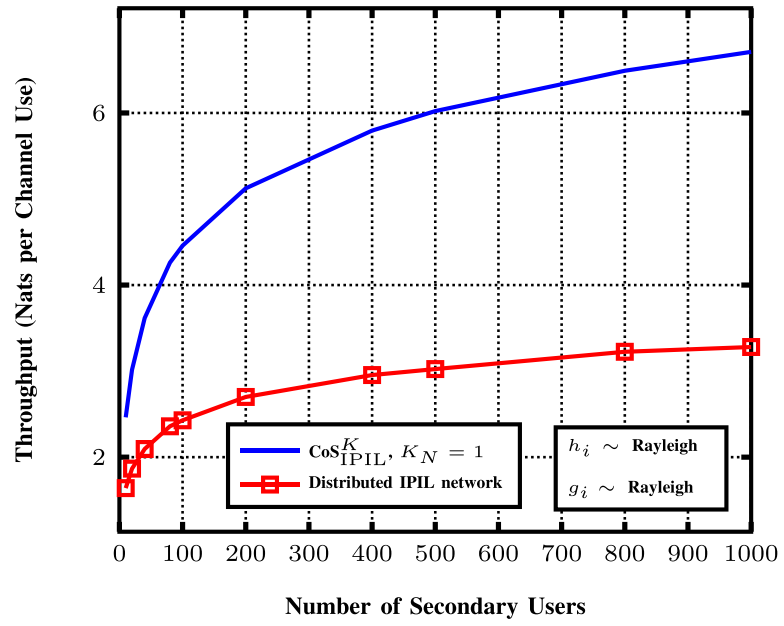

(b)

Fig. 4. Secondary network throughput scaling under $\operatorname{CoS}_{\mathrm{IL}}^{K}\left(K_{N}=1\right)$ and distributed IL network $(a)$. Secondary network throughput scaling under $\operatorname{CoS} K$ IPIL $\left(K_{N}=1\right)$ and distributed IPIL network $(b)$. Direct and interference channel gains are Rayleigh distributed in these figures. $P_{\text {ave }}$ and $Q_{\text {ave }}$ are set to $15 \mathrm{~dB}$ and $0 \mathrm{~dB}$, respectively.

Figure 2(d) depicts the dependence of the secondary network throughput under $\operatorname{CoS}_{\mathrm{IL}}^{\text {Full }}$ and $\operatorname{CoS}_{\mathrm{IL}}^{K}$ on $\gamma_{g}$ in a cognitive uplink with $N=50$. In this figure, the curves with $K_{N}=N$ represent the secondary network throughput under $\operatorname{CoS}_{\mathrm{IL}}^{\mathrm{Full}}$, and the curves with $K_{N}=1$ represent the secondary network throughput under $\operatorname{CoS}_{\mathrm{IL}}^{K}$. Direct channel gains are Rayleigh distributed, and interference channel gains are Weibull- $c$ and Nakagami- $m$ distributed. In Fig. 2(d), as $\gamma_{g}$ increases, interference channel gains become large, and SUs reduce their transmission powers to meet the interference constraint. Thus, the secondary network throughput drops as discussed above.

We demonstrate the throughput scaling behavior of the secondary network under $\operatorname{CoS}_{\mathrm{IPIL}}^{\mathrm{Full}}$ and $\operatorname{CoS}_{\mathrm{IPIL}}^{K}$ as a function of the number of SUs in Fig. 3. In this figure, the curves with $K_{N}=N$ represent the secondary network throughput under $\operatorname{CoS}_{\text {IPIL }}^{\text {Full }}$, and the curves with $K_{N}=1$ represent the secondary network throughput under $\operatorname{CoS}_{\mathrm{IPIL}}^{K} \cdot P_{\mathrm{ave}}$ and $Q_{\mathrm{ave}}$ are set to $15 \mathrm{~dB}$ and $0 \mathrm{~dB}$, respectively. Similar qualitative behavior continues to hold for other values of $P_{\text {ave }}$ and $Q_{\text {ave }}$.

Figure 3(a) illustrates the secondary network throughput scaling when direct channel gains are distributed according to the Rayleigh fading model, and interference channel gains are distributed according to the Weibull $-c$ fading model with $c=1.5$. Figure $3(\mathrm{a})$ indicates that the throughput of the secondary network scales according to $\log (N)$ for $c \leq 2$ as predicted by Theorem 2 . The $\log (N)+\log \left(P_{\text {ave }}\right)$ curve 


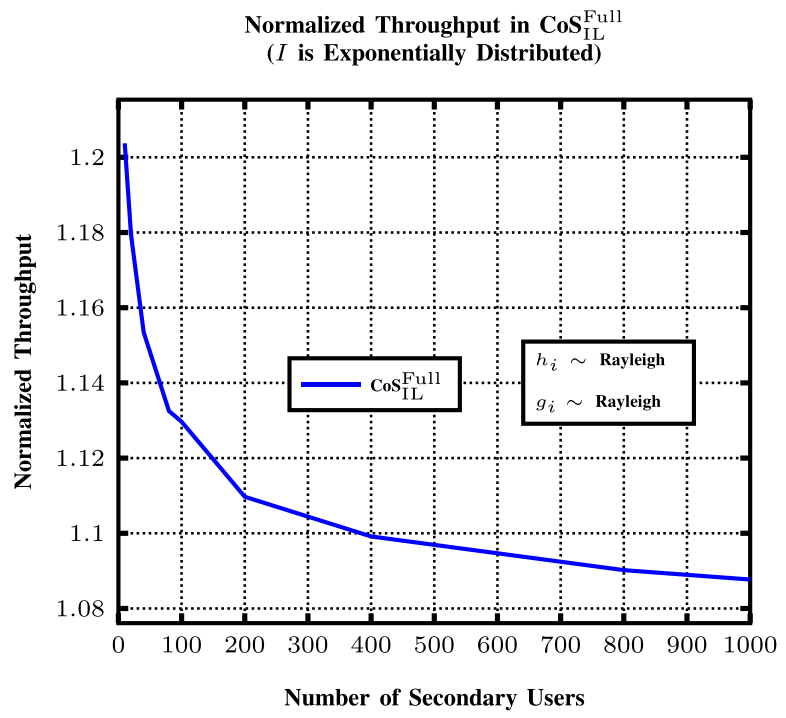

(a)

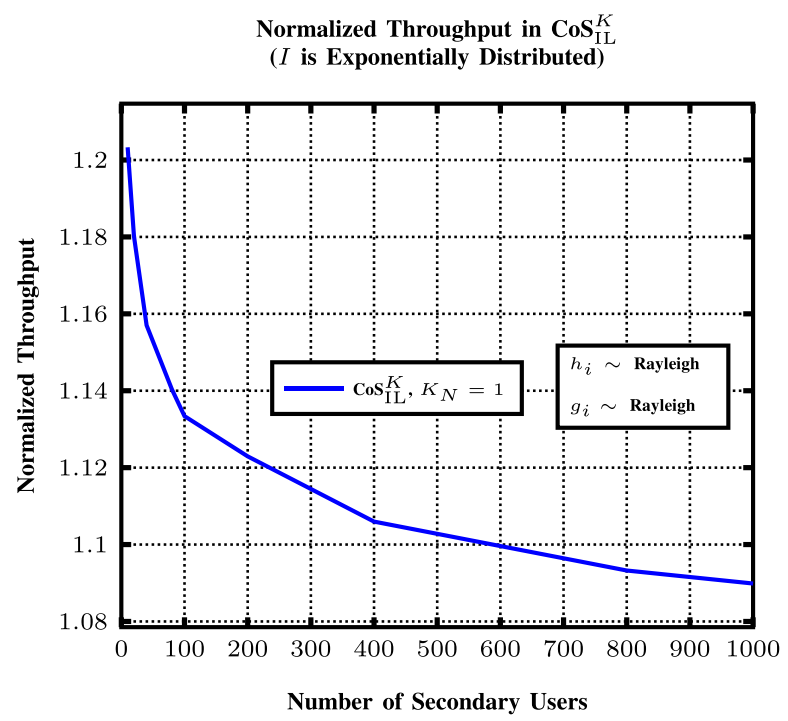

(b)

Fig. 5. Normalized throughput in $\operatorname{CoS}_{\mathrm{IL}}^{\mathrm{Full}}$ and $\operatorname{CoS}_{\mathrm{IL}}^{K}\left(K_{N}=1\right)$ when the primary interference power at the SBS is an exponentially distributed random variable with unit mean. The direct and interference channel gains are Rayleigh distributed. $P_{\text {ave }}$ and $Q_{\text {ave }}$ are set to $15 \mathrm{~dB}$ and $0 \mathrm{~dB}$, respectively.

represents the scaling behavior of the primary multiple access channel with individual power constraints obtained by removing the interference power constraint from the original IPIL network. Closeness of this curve to our simulated data rates confirms that an IPIL network behaves similar to a primary multiple access channel with individual power constraints for $\gamma_{g}<1$.

Figure 3(b) represents the secondary network throughput when direct channel gains are distributed according to the Rayleigh fading model, and interference channel gains are distributed according to the Weibull- $c$ fading model with $c=2.5$. As this figure shows, the secondary network throughput scales according to $\frac{2}{c} \log (N)$, which is also in accordance with the MDGs predicted by Theorem 2. In Fig. 3(b), the $0.8 \log (N)+\frac{1}{\gamma_{g}} \log \left(\eta_{g} \mathrm{E}\left[h^{\gamma_{g}}\right]\right)$ curve quantifies the throughput scaling behavior of the IL network obtained by removing the individual power constraints from the original IPIL network. Closeness of this curve to our simulated data rates confirms that an IPIL network behaves similar to an IL network for $\gamma_{g}>1$. Finally, Figs. 3(a)-(b) show that the throughput loss arising from implementing the $K$-out-of- $N$ feedback protocol is within one nat per channel use again when compared to the full primary cooperation scenario, which signifies that the $K$-out-of- $N$ feedback protocol is an effective primary-secondary feedback reduction policy in IPIL networks even for finitely many SUs, too.

Figure 4 shows the secondary network throughput scaling under $\operatorname{CoS}_{\mathrm{IL}}^{K}\left(K_{N}=1\right)$ and a fully distributed IL network (Fig. 4(a)), and under $\operatorname{CoS}_{\text {IPIL }}^{K}\left(K_{N}=1\right)$ and a fully distributed IPIL network (Fig. 4(b)). In this figure, direct and interference channel gains are Rayleigh distributed. $P_{\text {ave }}$ and $Q_{\text {ave }}$ are set to $15 \mathrm{~dB}$ and $0 \mathrm{~dB}$, respectively, in Fig. 4. As this figure shows, a cognitive uplink under $K$-out-of- $N$ feedback protocol with $K_{N}=1$ can achieve a substantially higher throughput when compared to that under the fully distributed implementation.
This observation also provides a numerical confirmation that the $K$-out-of- $N$ feedback protocol is a much more efficient solution for the primary-secondary feedback problem than the fully distributed implementation of cognitive uplinks when there is an opportunity to establish a very low-rate feedback link between the PBS and SBS.

We conclude this section by studying the throughput performance of secondary networks when the primary interference power at the SBS is a random variable. To elucidate this point clearly, we introduce the notion of normalized throughput for a secondary network in Fig. 5, which is defined to be the ratio of the secondary network throughput when $I$ is zero to that when $I$ is modeled as a random variable. By taking $I$ as an exponentially distributed random variable with unit mean, Fig. 5 clearly shows that the normalized throughput in both cases of $\operatorname{CoS}_{\mathrm{IL}}^{\text {Full }}$ and $\operatorname{CoS}_{\mathrm{IL}}^{K}\left(K_{N}=1\right)$ approaches one as the number of SUs increases. This numerical observation is inline with our theorems in Section V and confirms that the throughput scaling behavior of the secondary network remains unchanged when the primary's interference power at the SBS is modelled as a positive random variable with finite mean.

\section{Conclusions and Future Research Directions}

In this paper, we have studied the achievable throughput performance of the cognitive uplink under limited primary cooperation. To cope with the primary-secondary feedback link limitations, we have proposed a feedback protocol, named $K$-out-of- $N$ feedback protocol, in which the SBS only receives the $K_{N}$ smallest interference channel gains from the PBS. Under the optimum power control policy, it was shown that the $K$-out-of- $N$ feedback protocol achieves the optimum throughput scaling behavior for $K_{N}=O$ (1) in IL and IPIL networks, whereas it achieves the optimum throughput scaling behavior for $K_{N}=N^{\delta}$ with $\delta \in(0,1)$ in TPIL networks. Moreover, it was shown that the secondary network throughput (under both 
full and limited primary cooperation) scales scales according to $\frac{1}{\gamma_{g}} \log (N), \min \left(1, \frac{1}{\gamma_{g}}\right) \log (N)$ and $\frac{1}{n_{h}} \log \log (N)$ in IL, IPIL and TPIL networks, respectively. Here, $\gamma_{g}$ and $\frac{1}{n_{h}}$ are two parameters obtained from the distributions of interference and direct channel power gains, respectively. Our results shed light on the effect of fading distributions on the MDGs in the cognitive uplink. They also show that the optimum throughput scaling behavior can be attained in a cognitive uplink even with reduced primary-secondary cooperation.

The results presented in this paper can be extended in several important ways. One research direction of interest to generalize these results is to obtain similar scaling results for other performance measures such as outage and delay-limited capacities under appropriate operating conditions. Another important future research direction of interest is the extension of these results to multi-band and multi-antenna systems. Even though the same principles and asymptotic analysis techniques can be utilized for deriving the secondary network throughput scaling behavior, the optimum power and resource allocation strategies cannot be necessarily described in a closed form solution as in (3) or (5), which will potentially complicate the analysis for such extensions.

\section{APPENDIX A}

\section{Asymptotic Behavior of EXTREME ORder Statistics}

In this appendix, we study the concentration behavior of the extreme order statistic of a sequence of i.i.d. random variables as the number of elements in the sequence grows large. Later, this result will play a central role in deriving the cognitive radio throughput scaling behavior in different communication scenarios. To this end, let $\left\{Y_{i}\right\}_{i=1}^{N}$ be a sequence of i.i.d random variables with a common probability distribution function $F_{Y}$. We assume that $\lim _{y \rightarrow \infty} F_{Y}(y)=1, F_{Y}(y)<1$ for $y<\infty$, and there exists $y_{0}<\infty$ such that $F_{Y}\left(y_{1}\right)<F_{Y}\left(y_{2}\right)$ whenever $y_{0}<y_{1}<y_{2}<\infty$. We call a CDF possessing these properties an eventually increasing CDF. Let $G(y)$ be a function such that $\lim _{y \rightarrow \infty} G(y)\left(1-F_{Y}(y)\right)=1$. We say $G$ characterizes the tail behavior of $F_{Y}$. Without loss of generality, we assume that $G:(C, \infty) \mapsto \mathbb{R}_{+}$, and $G$ is strictly increasing on $(C, \infty)$. Note that $G$ is invertible, and we denote its functional inverse as $G^{-1} \cdot{ }^{3}$ Let $Y_{N}^{\star}$ be the extreme order statistic of $\left\{Y_{i}\right\}_{i=1}^{N}$, i.e., $Y_{N}^{\star}=\max _{1 \leq i \leq N} Y_{i}$. Let $F_{Y_{N}^{\star}}$ be the $\mathrm{CDF}$ of $Y_{N}^{\star}$.

The next lemma establishes an important concentration property for $Y_{N}^{\star}$. This result will be used to study the convergence behavior of $Y_{N}^{\star}$. It also shows that the asymptotic behavior of $Y_{N}^{\star}$ is characterized by $G$ as $N$ tends to infinity. Note that the class of eventually increasing distributions covers the class- $C$ distributions.

Lemma 2: Let $\left\{Y_{i}\right\}_{i=1}^{N}$ be a sequence of i.i.d. random variables with an eventually increasing common CDF $F_{Y}$ whose tail behavior is characterized by $G$. Also,

\footnotetext{
${ }^{3}$ Since $F_{Y}(y)$ is eventually increasing, $G(y)$ is also eventually increasing and tends to infinity as $y$ tends to infinity. Thus, we can find a large positive constant $C$ such that $G$ is strictly increasing on $(C, \infty)$, and $G$ is invertible on this interval.
}

let $Y_{N}^{\star}=\max _{1 \leq i \leq N} Y_{i}$. Then, for any $\epsilon$ belonging to $(0,1)$, we have $\lim _{N \rightarrow \infty} \operatorname{Pr}\left\{G^{-1}\left(N^{1-\epsilon}\right)<Y_{N}^{\star} \leq G^{-1}\left(N^{1+\epsilon}\right)\right\}=1$.

Proof: For $y$ large enough, we can express $F_{Y_{N}^{\star}}$ as $F_{Y_{N}^{\star}}(y)=\mathrm{e}^{-N \Theta\left(\frac{-1}{G(y)}\right)}$ since $\lim _{y \rightarrow \infty} G(y)\left(1-F_{Y}(y)\right)=1$. By using this expression for $F_{Y_{N}^{\star}}$, we have:

$$
\operatorname{Pr}\left\{Y_{N}^{\star} \leq G^{-1}\left(N^{1+\epsilon}\right)\right\}=\mathrm{e}^{-N \Theta\left(\frac{1}{N^{1+\epsilon}}\right)}=1-\Theta\left(\frac{1}{N^{\epsilon}}\right)
$$

and

$$
\operatorname{Pr}\left\{Y_{N}^{\star} \leq G^{-1}\left(N^{1-\epsilon}\right)\right\}=\mathrm{e}^{-N \Theta\left(\frac{1}{N^{1-\epsilon}}\right)}=\mathrm{e}^{-\Theta\left(N^{\epsilon}\right)}
$$

for all $\epsilon \in(0,1)$ and $N$ large enough. Therefore, we have $\operatorname{Pr}\left\{G^{-1}\left(N^{1-\epsilon}\right)<Y_{N}^{\star} \leq G^{-1}\left(N^{1+\epsilon}\right)\right\}=1-\Theta\left(\frac{1}{N^{\epsilon}}\right)$, which implies that $Y_{N}^{\star}$ lies in $\left[G^{-1}\left(N^{1-\epsilon}\right), G^{-1}\left(N^{1+\epsilon}\right)\right]$ with probability approaching 1 as $N$ grows large.

\section{APPENDIX B}

\section{THROUGHPUT SCALING FOR IL NETWORKS}

In this appendix, we first establish the secondary network throughput scaling behavior for $\operatorname{CoS}_{\mathrm{IL}}^{\text {Full }}$. Then, we will use this result to obtain an upper bound on the secondary network throughput in $\operatorname{CoS}_{\mathrm{IL}}^{K}$. In our derivations, without loss of generality, we take $W$ to be 1 for the sake of notational simplicity.

To this end, we need to study the asymptotic behavior of $X_{N}^{\star}\left(0, \mu_{N}\right)=\max _{1 \leq i \leq N} \frac{h_{i}}{\mu_{N} g_{i}}$ when $N$ grows large. We start our analysis by deriving the scaling behavior of $\tilde{R}(N, 0, \mu)=$ $\mathrm{E}\left[\log \left(X_{N}^{\star}(0, \mu)\right) 1_{\left\{X_{N}^{\star}(0, \mu) \geq 1\right\}}\right.$, where $\mu$ is a fixed positive constant. In the next lemma, we characterize the asymptotic tail behavior of the random variable $X_{i}(0, \mu)=\frac{h_{i}}{\mu g_{i}}$, where $X_{i}(\lambda, \mu)$ is defined as $X_{i}(\lambda, \mu)=\frac{h_{i}}{\lambda+\mu g_{i}}$. Then, we will use this lemma to establish the concentration behavior of $X_{N}^{\star}(0, \mu)$, and thereby to obtain the scaling behavior of $\tilde{R}(N, 0, \mu)$. Throughout this appendix, we assume that $h_{i}$ 's and $g_{i}$ 's are distributed according to $F_{h}$ and $F_{g}$, respectively, where $F_{h}$ and $F_{g}$ belong to the class- $C$ distributions.

Lemma 3: Let $F_{X_{i}(0, \mu)}$ be the CDF of $X_{i}(0, \mu)=\frac{h_{i}}{\mu g_{i}}$. Then, $\lim _{x \rightarrow \infty} \frac{1-F_{X_{i}(0, \mu)}(x)}{\xi x^{-\gamma g}}=1$, where $\xi=\frac{\eta_{g} \mathrm{E}\left[h_{i}^{\gamma_{g}}\right]}{\mu^{\gamma g}}$, and $\eta_{g}$ and $\gamma_{g}$ are positive constants derived from the behavior of the distribution function of $g_{i}$ near the origin.

Proof: See [27].

Now, we provide a key lemma that will enable us to upper and lower bound the secondary network scaling behavior in $\mathrm{CoS}_{\mathrm{IL}}^{\text {Full }}$.

Lemma 4: For $\mu>0$, we have $\lim _{N \rightarrow \infty} \frac{\tilde{R}(N, 0, \mu)}{\frac{1}{\gamma_{g}} \log (\tilde{\xi} N)}=1$.

Proof: Let $\tilde{X}_{N}^{\star}(0, \mu)=\frac{\log \left(X_{N}^{\star}(0, \mu)\right)}{\frac{1}{\gamma g} \log (\xi N)} 1_{\left\{X_{N}^{\star}(0, \mu) \geq 1\right\}}$, where $X_{N}^{\star}(0, \mu)=\max _{1 \leq i \leq N} X_{i}(0, \mu)$. It is sufficient to show that $\lim _{N \rightarrow \infty} \mathrm{E}\left[\tilde{X}_{N}^{\star}(0, \mu)\right]=1$. We start our analysis by proving that $\tilde{X}_{N}^{\star}(0, \mu) \stackrel{i . p .}{\longrightarrow} 1$ as $N$ tends to infinity, where $\stackrel{i . p .}{\longrightarrow}$ symbolizes convergence in probability. Using Lemma 3 , the tail behavior of $F_{X_{i}(0, \mu)}$ is characterized by 
$G(x)=\frac{x^{\gamma_{g}}}{\xi}$, where $\xi=\frac{\eta_{g} \mathrm{E}\left[h_{i}^{\gamma_{g}}\right]}{\mu^{\gamma_{g}}}$. Hence, $G^{-1}(x)=(\xi x)^{\frac{1}{\gamma_{g}}}$. Using Lemma 2, we have

$\operatorname{Pr}\left\{(\xi N)^{\frac{1}{\gamma_{g}}(1-\epsilon)}<X_{N}^{\star}(0, \mu) \leq(\xi N)^{\frac{1}{\gamma_{g}}(1+\epsilon)}\right\}=1-\Theta\left(\frac{1}{N^{\epsilon}}\right)$

which implies $\tilde{X}_{N}^{\star}(0, \mu) \stackrel{\text { i.p. }}{\longrightarrow} 1$ as $N$ grows large. Since convergence in probability does not always imply convergence in mean [31], we need to show that $\left\{\tilde{X}_{N}^{\star}(0, \mu)\right\}_{N=1}^{\infty}$ is uniformly integrable to complete the proof. We refer interested readers to [27] for the proof of uniform integrability.

The next lemma characterizes the asymptotic behavior of the Lagrange multiplier $\mu_{N}$ as $N$ becomes large. Later, we will use this result to provide upper and lower bounds for $\tilde{R}(N, 0, \mu)$. This result will also be helpful to conclude the logarithmic effect of $Q_{\text {ave }}$ on the secondary network throughput under $\operatorname{CoS}_{\mathrm{IL}}^{\text {Full }}$.

Lemma 5: Let $\mu_{N}$ be the Lagrange multiplier corresponding to the average interference power constraint in $\operatorname{CoS}_{\mathrm{II}}^{\text {Full }}$. Then, $\lim _{N \rightarrow \infty} \mu_{N}=\frac{1}{Q_{\text {ave }}}$.

Proof: First, we show that $\liminf _{N \rightarrow \infty} \mu_{N}>0$ by contradiction. Assume $\liminf _{N \rightarrow \infty} \mu_{N}=0$. This means that, for any given $\epsilon>0$, we can find a subsequence $\left\{N_{j}\right\}_{j=1}^{\infty}$ such that $\mu_{N_{j}} \leq \epsilon$ for $N_{j}$ large enough. The average interference power for $N_{j}$ large enough can be lower bounded as $\mathrm{E}\left[\left(\frac{1}{\mu_{N_{j}}}-\frac{1}{X_{N_{j}}^{\star}(0,1)}\right)^{+}\right] \geq \mathrm{E}\left[\left(\frac{1}{\epsilon}-\frac{1}{X_{N_{j}}^{\star}(0,1)}\right)^{+}\right]$. Note that $\left(\frac{1}{\epsilon}-\frac{1}{X_{N_{j}}^{\star}(0,1)}\right)^{+} \stackrel{\text { i.p. }}{\longrightarrow} \frac{1}{\epsilon}$. Applying Fatou's lemma, we have $\liminf _{N_{j} \rightarrow \infty} \mathrm{E}\left[\left(\frac{1}{\mu_{N_{j}}}-\frac{1}{X_{N_{j}}^{\star}(0,1)}\right)^{+}\right] \geq \frac{1}{\epsilon}$, which implies that the average interference power constraint will be violated when $N_{j}$ is large enough if we choose $\epsilon$ sufficiently small. Thus, we conclude that $\liminf _{N \rightarrow \infty} \mu_{N}>0$. Now, we will complete the proof by using the fact that $\mu_{N}$ cannot be arbitrarily close to zero as $N$ grows large. The average interference power constraint can be expressed as

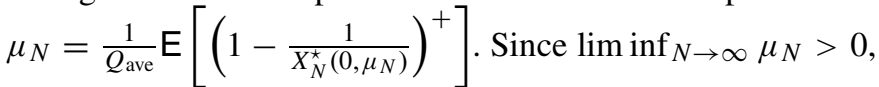
the desired result follows from the dominated convergence theorem. ${ }^{4}$

\section{A. Proof of Throughput Scaling in $\operatorname{CoS}_{I L}^{\text {Full }}$}

Now, we establish the secondary network throughput scaling behavior under the communication scenario $\operatorname{CoS}_{\mathrm{IL}}^{\text {Full }}$. First, we note that $\tilde{R}(N, 0, \mu)$ is a decreasing function of $\mu$. Therefore, for any given $\epsilon>0$, we can find a constant $N^{\prime}$ large enough such that $R_{\mathrm{IL}}(N)$ can be upper and lower bounded as

$$
\tilde{R}\left(N, 0, \frac{1+\epsilon}{Q_{\text {ave }}}\right) \leq R_{\mathrm{IL}}(N) \leq \tilde{R}\left(N, 0, \frac{1-\epsilon}{Q_{\text {ave }}}\right)
$$

for all $N \geq N^{\prime}$ since $\mu_{N}$ converges to $\frac{1}{Q_{\text {ave }}}$ as $N$ becomes large. Using Lemma 4, for any $\epsilon>0$ and $N$ large enough,

\footnotetext{
${ }^{4}$ Note that Lebesgue dominated convergence theorem remains valid if almost sure convergence is replaced with convergence in probability [32].
}

$R_{\mathrm{IL}}(N)$ can be further upper and lower bounded as

$$
\begin{gathered}
(1-\epsilon) \frac{1}{\gamma_{g}} \log \left(\left(\frac{Q_{\text {ave }}}{1+\epsilon}\right)^{\gamma_{g}} \eta_{g} \mathrm{E}\left[h^{\gamma_{g}}\right] N\right) \leq R_{\mathrm{IL}}(N) \\
\leq(1+\epsilon) \frac{1}{\gamma_{g}} \log \left(\left(\frac{Q_{\text {ave }}}{1-\epsilon}\right)^{\gamma_{g}} \eta_{g} \mathrm{E}\left[h^{\gamma_{g}}\right] N\right),
\end{gathered}
$$

where $h$ is a generic random variable with $\mathrm{CDF} F_{h}$. Thus, we have $\lim _{N \rightarrow \infty} \frac{R_{\mathrm{IL}}(N)}{\log (N)}=\frac{1}{\gamma_{g}}$.

\section{B. Proof of Throughput Scaling in $\operatorname{CoS}_{I L}^{K}$}

The secondary network throughput in $\operatorname{CoS}_{\mathrm{IL}}^{\text {Full }}$ serves as an upper bound for the secondary network throughput in $\mathrm{CoS}_{\mathrm{IL}}^{K}$, i.e., $R_{\mathrm{IL}}\left(K_{N}\right) \leq R_{\mathrm{IL}}(N)$, since more information is available at the SBS to perform power control and user scheduling under $\operatorname{CoS}_{\mathrm{IL}}^{\text {Full }}$. Thus, we have $\lim \sup _{N \rightarrow \infty} \frac{R_{\mathrm{IL}}\left(K_{N}\right)}{\log (N)} \leq \frac{1}{\gamma_{g}}$.

To prove the other direction, consider a sub-optimum power allocation policy $\hat{\boldsymbol{P}}_{K_{N}}^{\mathrm{IL}}(\boldsymbol{g})$, which only depends on interference channel power gains, such that the $i$ th $\mathrm{SU}$ transmits with power $\hat{P}_{i, K_{N}}^{\mathrm{IL}}(g)=\frac{Q_{\text {ave }}}{g_{i}} 1_{\left\{g_{i}=\min _{1 \leq j \leq K_{N}} g_{\pi(j)}\right\}}$. Note that $\min _{1 \leq i \leq K_{N}} g_{\pi(i)}=\min _{1 \leq i \leq N} g_{i}=g_{\min }(N)$. The average interference power at the PBS using $\hat{\boldsymbol{P}}_{K_{N}}^{\mathrm{IL}}$ is given by $\mathrm{E}\left[\sum_{i=1}^{N} \hat{P}_{i, K_{N}}^{\mathrm{IL}} g_{i}\right]=Q_{\mathrm{ave}}$. Thus, $\hat{\boldsymbol{P}}_{K_{N}}^{\mathrm{IL}}$ satisfies the average interference power constraint, and it is a feasible power allocation policy for a secondary network under $\operatorname{CoS}_{\mathrm{IL}}^{K}$. Let $\hat{R}_{\mathrm{IL}}\left(K_{N}\right)$ be the secondary network throughput achieved by using $\hat{\boldsymbol{P}}_{K_{N}}^{\mathrm{IL}}$. Plugging $\hat{\boldsymbol{P}}_{K_{N}}^{\mathrm{IL}}$ inside the Shannon rate formula and ignoring the constant $1, \hat{R}_{\mathrm{IL}}\left(K_{N}\right)$ can be lower bounded as $\hat{R}_{\mathrm{IL}}\left(K_{N}\right) \geq \log \left(Q_{\mathrm{ave}}\right)+\mathrm{E}[\log (h)]+\mathrm{E}\left[\log \left(\frac{1}{g_{\min }(N)}\right)\right]$,

where $h$ is a generic random variable with $\mathrm{CDF} F_{h}$. Using arguments similar to the ones used to prove Lemma 4, it is easy to show that $\lim _{N \rightarrow \infty} \frac{\mathrm{E}\left[\log \left(\frac{1}{g_{\min }(N)}\right)\right]}{\log (N)}=\frac{1}{\gamma_{g}}$ since $\frac{1}{g_{\min }(N)}=\max _{1 \leq i \leq N} \frac{1}{g_{i}}$ and the tail behavior of $\frac{1}{g_{i}}$ is characterized by $\lim _{x \rightarrow \infty} \frac{\operatorname{Pr}\left\{\frac{1}{g_{i}} \geq x\right\}}{\eta_{g} x^{-\gamma_{g}}}=1$. Also, we have $|\mathrm{E}[\log (h)]|<$ $\infty$. Thus, $\lim \inf _{N \rightarrow \infty} \frac{\hat{R}_{\mathrm{LL}}\left(K_{N}\right)}{\log (N)} \geq \frac{1}{\gamma_{g}}$. Since $\hat{\boldsymbol{P}}_{K_{N}}^{\mathrm{IL}}$ is a suboptimum power allocation policy for a secondary network under $\operatorname{CoS} \mathrm{IL}_{\mathrm{IL}}^{K}$, we have $\hat{R}_{\mathrm{IL}}\left(K_{N}\right) \leq R_{\mathrm{IL}}\left(K_{N}\right)$ and therefore $\lim \inf _{N \rightarrow \infty} \frac{R_{\mathrm{LL}}\left(K_{N}\right)}{\log (N)} \geq \frac{1}{\gamma_{g}}$, which completes the proof.

\section{ACKNOWLEDGEMENTS}

The authors thank H. Vincent Poor for his reading the earlier versions of this work and providing encouraging feedback.

\section{REFERENCES}

[1] S. Haykin, "Cognitive radio: Brain-empowered wireless communications," IEEE J. Sel. Areas Commun., vol. 23, no. 2, pp. 201-220, Feb. 2005.

[2] A. Goldsmith, S. A. Jafar, I. Maric, and S. Srinivasa, "Breaking spectrum gridlock with cognitive radios: An information theoretic perspective," Proc. IEEE, vol. 97, no. 5, pp. 894-914, Apr. 2009.

[3] I. F. Akyildiz, W.-Y. Lee, M. C. Vuran, and S. Mohanty, "NeX generation/dynamic spectrum access/cognitive radio wireless networks: A survey," Comput. Netw., vol. 50, no. 13, pp. 2127-2159, Sep. 2006. 
[4] A. Ghasemi and E. S. Sousa, "Fundamental limits of spectrum-sharing in fading environments," IEEE Trans. Wireless Commun., vol. 6, no. 2, pp. 649-658, Feb. 2007.

[5] L. Musavian and S. Aïssa, "Capacity and power allocation for spectrumsharing communications in fading channels," IEEE Trans. Wireless Commun., vol. 8, no. 1, pp. 148-156, Jan. 2009.

[6] J. M. Peha, "Approaches to spectrum sharing," IEEE Commun. Mag., vol. 43, no. 2, pp. 10-12, Feb. 2005.

[7] D. W. K. Ng, E. S. Lo, and R. Schober, "Energy-efficient resource allocation in multi-cell OFDMA systems with limited backhaul capacity," IEEE Trans. Wireless Commun., vol. 11, no. 10, pp. 3618-3631, Oct. 2012

[8] A. Sanderovich, O. Somekh, H. V. Poor, and S. Shamai (Shitz), "Uplink macro diversity of limited backhaul cellular network," IEEE Trans. Inf. Theory, vol. 55, no. 8, pp. 3457-3478, Aug. 2009.

[9] O. Simeone, O. Somekh, H. V. Poor, and S. Shamai (Shitz), "Local base station cooperation via finite-capacity links for the uplink of linear cellular networks," IEEE Trans. Inf. Theory, vol. 55, no. 1, pp. 190-204, Jan. 2009.

[10] E. Nekouei, H. Inaltekin, and S. Dey, "Power control and asymptotic throughput analysis for the distributed cognitive uplink," IEEE Trans. Commun., vol. 62, no. 1, pp. 41-58, Jan. 2014.

[11] G. L. Stüber, Principles of Mobile Communication. Boston, MA, USA: Kluwer, 1996.

[12] M. K. Simon and M.-S. Alouini, Digital Communication Over Fading Channels, 2nd ed. Hoboken, NJ, USA: Wiley, 2005.

[13] J. C. F. Li, W. Zhang, and J. Yuan, "Opportunistic spectrum sharing in cognitive radio networks based on primary limited feedback," IEEE Trans. Commun., vol. 59, no. 12, pp. 3272-3277, Dec. 2011.

[14] Z. Wang and W. Zhang, "Spectrum sharing with limited channel feedback," IEEE Trans. Wireless Commun., vol. 12, no. 5, pp. 2524-2532, May 2013.

[15] Z. Wang and W. Zhang, "Exploiting multiuser diversity with 1-bit feedback for spectrum sharing," IEEE Trans. Commun., vol. 62, no. 1, pp. 29-40, Jan. 2014.

[16] J.-P. Hong and W. Choi, "Throughput characteristics by multiuser diversity in a cognitive radio system," IEEE Trans. Signal Process., vol. 59, no. 8, pp. 3749-3763, Aug. 2011.

[17] T. W. Ban, W. Choi, B. C. Jung, and D. K. Sung, "Multi-user diversity in a spectrum sharing system," IEEE Trans. Wireless Commun., vol. 8, no. 1, pp. 102-106, Jan. 2009.

[18] T. W. Ban, D. K. Sung, B. C. Jung, and W. Choi, "Capacity analysis of an opportunistic scheduling system in a spectrum sharing environment," in Proc. IEEE Global Telecommun. Conf., New Orleans, LA, USA, Nov./Dec. 2008, pp. 1-5.

[19] R. Zhang and Y.-C. Liang, "Investigation on multiuser diversity in spectrum sharing based cognitive radio networks," IEEE Commun. Lett., vol. 14, no. 2, pp. 133-135, Feb. 2010.

[20] T.-W. Ban and B. C. Jung, "On the multi-user diversity with fixed power transmission in cognitive radio networks," IEEE Wireless Commun. Lett., vol. 3, no. 1, pp. 74-77, Feb. 2014

[21] E. Nekouei, H. Inaltekin, and S. Dey, "Throughput scaling in cognitive multiple access with average power and interference constraints," IEEE Trans. Signal Process., vol. 60, no. 2, pp. 927-946, Feb. 2012.

[22] H. Wang, J. Lee, S. Kim, and D. Hong, "Capacity of secondary users exploiting multispectrum and multiuser diversity in spectrumsharing environments," IEEE Trans. Veh. Technol., vol. 59, no. 2, pp. 1030-1036, Feb. 2010.

[23] A. Tajer and X. Wang, "Multiuser diversity gain in cognitive networks," IEEE/ACM Trans. Netw., vol. 18, no. 6, pp. 1766-1779, Dec. 2010.

[24] Y. Li and A. Nosratinia, "Capacity limits of multiuser multiantenna cognitive networks," IEEE Trans. Inf. Theory, vol. 58, no. 7, pp. 4493-4508, Jul. 2012

[25] D. Tse and P. Viswanath, Fundamentals of Wireless Communication. Cambridge, U.K.: Cambridge Univ. Press, 2005.

[26] E. Nekouei, H. Inaltekin, and S. Dey, "Asymptotically optimal channel feedback protocol design for cognitive multiple access channels," in Proc. IEEE Global Commun. Conf., Anaheim, CA, USA, Dec. 2012, pp. 2196-2202.

[27] E. Nekouei, H. Inaltekin, and S. Dey. (Sep. 2015). "Multiuser diversity for the cognitive uplink with generalized fading and reduced primary's cooperation," Dept. Elect. Electron. Eng., Univ. Melbourne, Melbourne, VIC, Australia, Tech. Rep. [Online]. Available: http://arxiv.org/abs/1209.1424

[28] Y. S. Soh, T. Q. S. Quek, M. Kountouris, and G. Caire, "Cognitive hybrid division duplex for two-tier femtocell networks," IEEE Trans. Wireless Commun., vol. 12, no. 10, pp. 4852-4865, Oct. 2013.
[29] A. De Domenico, E. C. Strinati, and M.-G. Di Benedetto, "A survey on MAC strategies for cognitive radio networks," IEEE Commun. Surveys Tuts., vol. 14, no. 1, pp. 21-44, 1st Quart. 2012.

[30] J. G. Andrews, "Seven ways that HetNets are a cellular paradigm shift," IEEE Commun. Mag., vol. 51, no. 3, pp. 136-144, Mar. 2013.

[31] P. Billingsley, Probability and Measure, 3rd ed. New York, NY, USA: Wiley, 1995

[32] V. I. Bogachev, Measure Theory, vol. 1. New York, NY, USA: Springer, 2007.

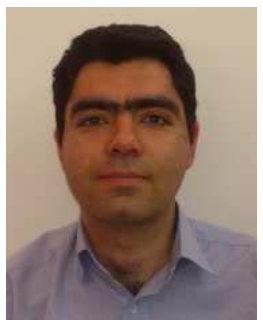

Ehsan Nekouei (S'11-M'14) received the B.Sc. degree from the Shahid Bahonar University of Kerman, Kerman, Iran, in 2003, the M.Sc. degree from Tarbiat Modares University, Tehran, Iran, in 2006, and the Ph.D. degree in electrical engineering from the University of Melbourne, in 2013. He is currently a Research Fellow with the Department of Electrical and Electronic Engineering, University of Melbourne. His research interests include game theory, information theory, and dynamical systems.

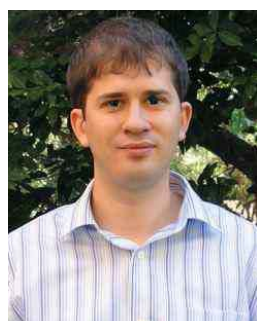

Hazer Inaltekin (S'04-M'06) received the B.S. (Hons.) degree in electrical and electronics engineering from Boğaziçi University, Istanbul, Turkey, in 2001, and the M.S./Ph.D. degree in electrical and computer engineering from Cornell University, Ithaca, NY, USA, in 2006. He was a Post-Doctoral Research Associate with Cornell University from 2006 to 2007, and Princeton University, Princeton, NJ, USA, from 2007 to 2009. In 2009, he joined the Department of Electrical and Electronic Engineering, University of Melbourne, as a Research Fellow. He was a Senior Research Fellow with the Department of Electrical and Electronic Engineering in 2011. Since 2011, he has been a Faculty Member with Antalya International University, where he is currently an Associate Professor of electrical and electronics engineering. His research interests include wireless communications, wireless networks, social networks, game theory, and information theory.

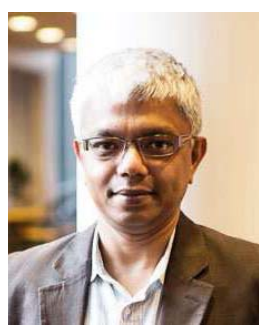

Subhrakanti Dey (M'96-SM'06) was born in India in 1968. He received the B.Tech. and M.Tech. degrees from the Department of Electronics and Electrical Communication Engineering, IIT Kharagpur, in 1991 and 1993, respectively, and the Ph.D. degree from the Department of Systems Engineering, Research School of Information Sciences and Engineering, Australian National University, Canberra, in 1996

He was a Post-Doctoral Research Fellow with the Department of Systems Engineering, Australian National University, from 1995 to 1997 and from 1998 to 2000. From 1997 to 1998, he was a Post-Doctoral Research Associate with the Institute for Systems Research, University of Maryland, College Park. He was a Professor with the Department of Electrical and Electronic Engineering, University of Melbourne, Parkville, Australia, from 2000 to 2013. He is currently a Professor with the Department of Engineering Sciences, Uppsala University, Sweden. His current research interests include networked control systems, wireless communications and networks, signal processing for sensor networks, and stochastic and adaptive signal processing and control.

Prof. Dey serves on the Editorial Board of the IEEE Transactions on Signal Processing and the IEEE Transactions on Control of Network Systems. He was also an Associate Editor of the IEEE Transactions on Signal Processing from 2007 to 2010, the IEEE Transactions on Automatic Control from 2004 to 2007, and Systems and Control Letters (Elsevier) from 2003 to 2013 